\title{
Income taxes, subsidies to education, and investments in human capital
}

\author{
Concetta Mendolicchio \\ IAB \\ and \\ Dimitri Paolini \\ DEIR and CRENoS, Università di Sassari \\ and \\ Tito Pietra ${ }^{1}$ \\ DSE, Università di Bologna
}

Version: January 24, 2010

We study a two-sector economy with investments in human and physical capital and imperfect labor markets. Human and physical capital are heterogeneous. Workers and firms endogenously select the sector they are active in, and choose the amount of their sector-specific investments in human and physical capital. To enter the high-skill sector, workers must pay a fixed cost that we interpret as direct cost of education. Given the distribution of the agents across sectors, at equilibrium, in each sector there is underinvestment in both human and physical capital, due to non-contractibility of investments. A second source of inefficiency is related to the self-selection of the agents into the two sectors. It typically induces too many workers to invest in education. Under suitable restrictions on the parameters, the joint effect of the two distortions is that equilibria are characterized by too many people investing too little effort in the high skill sector.

We also analyze the welfare properties of equilibria and study the effects of several tax-subsidy policies on the total expected surplus.

\section{JEL classification: J24; $\mathrm{H} 2$}

Keywords: Human capital; Efficiency; Labour income tax

Corresponding author: T. Pietra, Tel.: +39051 2098026; fax +39051 2098040. E-mail address: tito.pietra@unibo.it

\footnotetext{
${ }^{1} \mathrm{~A}$ previous version of the paper has been circulated as "Human capital policies in a static, two-sector economy with imperfect markets", CRENoS 08-07. The paper is part of the Ph.D. dissertation at IRES, Universitè Catholique de Louvain, of the first author. We thank for helpful comments the members of her dissertations committee (R. Boucchekine, V. Vandenberghe, M. Belot, B. Decreuse, B. Van der Linden), P. Pestieau, and participants of a seminars at the University of Luxembourg, at the Università di Salerno and at ASSET 2009. The usual disclaimers apply. We acknowledge the financial support of MIUR-PRIN 2006 and the Fondazione Banco di Sardegna. The first author acknowledges the support of the "Programma Visiting Professor" of the Università di Sassari (Italy).
} 


\section{INTRODUCTION}

Causes and consequences of investments in human capital have been a central field of research in the last few decades for several motivations. Among them, the relevance of human capital externalities in growth theory, and the issues related to the dynamics of the wage premium and, more generally, to the evolution of income distribution. Still, the analysis of human capital externalities is far from settled from both the empirical and the theoretical viewpoints. Empirically, it is not obvious that there are significant, positive differences between social and private returns, at least at the level of subsidies prevailing in most Western countries. ${ }^{2}$ From a theoretical viewpoint, the exact microeconomic mechanism generating the externality is not fully understood. A better understanding of its nature has policy relevance. This is true even if one is willing to take for granted that there are no significant, unexploited, positive externalities, because this is typically obtained with high subsidies to education. ${ }^{3}$

In this paper, we extend the microeconomic analysis of the externalities related to investments in human capital and derive some results on the welfare effects of several policies: fixed tax/subsidies on the direct cost of the acquisition of high skill human capital, and tax/subsidies on labor income, or - equivalently in our set-up on the investment in human capital.

We consider economies with three key features:

1. Workers are ex-ante heterogeneous, while firms are not,

2. Investments in human and physical capital are non-contractible,

3. There are two separate sectors employing different kinds of human and physical capital, so that an agent must choose both the level of his/her investment and its type.

The economy is basically a two-sector generalization, with sector specific inputs, of the model considered in Acemoglu (1996). In his framework, firms and workers choose the amount of their investments. Then, they are matched randomly, and income distribution is determined by a bargaining process. After agents have chosen the sector they are going to be active in (hence, the type of their investment), the model considered here reduces to a pair of separated Acemoglu's economies. In our set-up, income distribution takes place through bargaining, too. However, bear in mind that, when workers are heterogeneous, the crucial feature is noncontractibility of investments: the bargaining set-up affects several details, but not some key aspects of the welfare results.

Our main departure from Acemoglu (1996) is that we adopt the notion of human capital put forth in Roy (1951): there are distinct markets for high skill and low skill labor, that we assume to be perfectly non substitutable (hence, the twosector structure). However, contrary to what is often assumed in Roy models, once a worker has selected the type of human capital she wants to acquire, she still has

\footnotetext{
${ }^{2}$ For the U.S.A., a negative conclusion is reached, for instance, by Heckman, Layne-Farrar, and Todd (1996) and by Acemoglu and Angrist (2001). For E.U. countries, the results in De la Fuente (2003) are also negative. See also Krueger and Lindhal (2000).

${ }^{3}$ In 2005, in the OECD average, $85.5 \%$ of the direct cost of education (all levels included) is financed by public sources (see OECD (2008, Table B3.1, p. 251)). The EU19 average is $90.5 \%$. At the tertiary level, these percentages are, respectively, $73.1 \%$ and $82.5 \%$ (Table B3.2b, p. 253).
} 
to decide how much effort to invest. As common in the literature, human capital translates one-to-one into efficiency units of high skill (low skill, respectively) labor. ${ }^{4}$ Most of the recent literature takes a different point of view, adopting the efficiency units approach with homogeneous human capital, therefore ruling out, by assumption, all the consequences of self-selection, which are, instead, relevant from both the theoretical and the empirical viewpoints. ${ }^{5}$

With imperfect markets and self-selection of the agents into different markets, two distinct distortions are at work. In the main text, lack of contractibility of investments and the bargaining set-up generate an hold-up problem, inducing an inefficiently low level of investments, in human and physical capital of both types (hence, in each sector). Secondly, due to lack of contractibility, agents choose type and level of their investments looking at the distribution of their expected future returns, which, in turn, depends upon the distribution of the investments of the potential partners in each sector. Given that workers are heterogeneous, a switch of a subset of them from one sector to the other affects the distribution of returns of the firms, hence their optimal investments. This second potential source of distortion is independent of the random matching-bargaining set-up, and is at work even when spot labour markets are perfectly competitive. ${ }^{6}$ Consequently, public policies have two distinct effects on total surplus, the index of welfare adopted here. The first is their direct impact on the optimal investments of the agents acquiring a sectorspecific asset: we will refer to it as incentive effect. The second is the one on the agents' distribution across markets: we call it composition effect, following Charlot and Decreuse (2005) (see also Decreuse (2008)). In "pure" Roy models (with selfselection, but no choice of the investment effort) only the composition effect is at play. In "pure" efficiency-units models (without self-selection) only the incentive effect is at work. Our model allows us to study the interaction between the two phenomena.

We consider two separate sectors, using sector specific inputs (high/low skill capital). The crucial property is that human and physical capital are heterogeneous. To identify one type of capital with one sector somewhat simplifies the set-up and sharpens the welfare results. However, the two distinct distortions would be at work even with just one productive sector employing both high and low skill labor. Bear in mind that, whenever in the sequel we mention the two-sector structure of the economy, we implicitly mean that the two sectors use different kinds of human and physical capital.

Some of the results in Acemoglu (1996) survive in our set-up. For instance, in both cases, the human capital externality is related to its (sector-specific) average

\footnotetext{
${ }^{4}$ As usual, we can also interpret effort as elastic supply of labor of a given skill.

${ }^{5}$ A survey supporting this claim is in Sattinger (1993). For more recent discussions of the different empirical implications of efficiency units vs. Roy models see, for instance, Carneiro, Heckman, and Vytlacil (2001). Investments in human capital in a two-sector economy with frictions due to random matching (but with perfectly inelastic supply of human and physical capital) have been studied in Sattinger (2003), Charlot and Decreuse (2005), and Mendolicchio, Paolini, and Pietra (2008).

${ }^{6}$ This case is briefly analyzed in Appendix 2, where, we consider economies with perfectly competitive spot labor markets, non-contractibility of investments, and asymmetric information. Due to perfect competition, the hold-up problem disappears, and (taking as given the distribution of the agents across sectors) investments are at their constrained efficient level. However, due to asymmetric information and lack of contractibility, the composition effect still induces constrained inefficiency of equilibria, which are always characterized by overinvestment in education.
} 
level, not to its aggregate level (as postulated in Lucas (1988)). There are, instead, sharp differences with respect to the policy prescriptions: in the one-sector model, subsidies to investments in human capital (or to labor supply) are unambiguously beneficial. This is because only the incentive effect is at play: a subsidy to the investments in human capital of any subset of agents increases them and, therefore, their expected value as a first order effect. This has a positive impact on the firms' investment decisions and, in turn, further increases the optimal investment of all the workers. This chain of positive feedbacks guarantees that these subsidies are (in a neighborhood of zero) welfare improving. To reformulate the point differently: in one-sector economies, there is a unique distortion induced by the hold-up problem which induces underinvestment for both firms and workers. Any policy increasing the investments of any subset of agents is welfare improving.

With two sectors, the incentive effect of a policy can be strengthened, weakened, or overturned, by its composition effect. In the final section of the paper, we study the welfare effects of several kinds of balanced budget policies based on $\operatorname{tax}$ /subsidies to the direct costs of education, and on skill-contingent subsidies to labor supply. Consider, for instance, subsidies to low skilled labor income (or a lower marginal tax rate for low incomes) financed with lump-sum taxes. If total factor productivities are sufficiently diverse across sectors, they always increase total surplus, because their positive effect on individual effort in this sector is strengthened by the composition effect, i.e., by the improvement of the expected quality of the pool of workers in both markets. An increase in taxes on the direct costs of education (again, balanced with lump-sum taxes) also increases total surplus, because of its composition effect. On the other hand, subsidies to high skill labor incomes have a (first order) positive incentive effect on the investments of these workers, but a negative composition effect. Subsidies to the investments in the high-skill sector have always a negative impact on the equilibrium utility of low-skilled workers (and on the equilibrium profits of the firms active in that sector). The total effect for agents active in the high-skill sector may be positive or negative, according to the magnitudes of the (positive) incentive effect and the (negative) composition effect. We provide a robust example where the total effect of these subsidies on surplus is negative.

There is a large literature on the effects of subsidies to education and of labor income taxes on accumulation of human capital. The usual arguments favoring subsidies hinge either on their positive externality effects, or on the existence of liquidity constraints. Additionally, subsidies to education have been analyzed as one of the components of the optimal mix of redistributive policies (see Bovenberg and Jacobs (2005), Jacobs (2005, 2007), Jacobs and Bovenberg (2008), Jacobs, Schindler and Yang (2009), Schindler and Weigert (2008, 2009)). The last two aspects may be both empirically and theoretically important, but we abstract from them, focussing the analysis on the pure efficiency issue related to the presence of an hold-up problem and of self-selection.

The classical analysis of the effects of labor income tax on investments in human capital started with the seminal papers by Ben-Porath (1970), Boskin (1975) and Heckman (1976)..$^{7}$ A flat labor income tax has a negative impact on human capital accumulation just because of non-deductibility of the direct costs of education. On

\footnotetext{
${ }^{7}$ As mentioned above, in our set-up, one obtains substantially identical results considering direct (non-linear) subsidies to effort and subsidies to the direct costs of education. Previous, related work in this area includes Blankenau (2005), Blankenau and Camera (2006, 2009), Caucutt and Kumar (2003), Lloyd-Ellis (2000), Sahin (2004), and Su (2004).
} 
the other hand, by depressing the net interest rate, in fully specified life-cycle models of consumer behavior, a tax on total income may actually have a positive effect. Eaton and Rosen (1980) extend the analysis to (uninsurable) multiplicative wage uncertainty, pointing out that a flat earning tax affects investments in human capital through its effects on their riskiness and (via an income effect) on the attitude toward risk (see, also, Anderberg and Andersson (2003), and Anderberg (2008)). Consider now a progressive income tax (compared with a revenue-neutral flat one). The canonical conclusion is that it discourages investments at the high skill level, while it may encourage them for the less skilled. While the literature provides us with many insights, it mostly deals with economies where there is no self-selection into different skills, so that one of the key mechanism at work in our economy is absent. Also, bear in mind that, in our set-up, at the equilibrium, workers face no uncertainty, so that the mechanism pointed out in Eaton and Rosen (1980) is absent.

The structure of the paper is the following. Section 2 discusses the general features of the model. Section 3 and 4 analyze the benchmark, Walrasian, economy, and the one with imperfect labor markets. Section 5 studies the welfare properties of the equilibria of the economy with frictions. Most of the details are in Appendix 1. In Appendix 2, we sketch the analysis of a perfectly competitive economy with asymmetric information and self-selection into the two sectors.

\section{THE MODEL}

The economy is composed by two separate production sectors, denoted by $s \in$ $\{n e, e\}$. Workers (denoted by a subscript $i$ when we refer to individuals, $I$ when we refer to their set) and firms (denoted by $j$ and $J$, respectively) can choose to enter one of the two sectors, paying a fixed cost. Workers' costs, $\left(c_{I}^{n e}, c_{I}^{e}\right)$, are exogenous, and can be interpreted as private, direct, fixed costs of education (tuitions and the like). We denote firms' costs $\left(d_{J}^{n e}, d_{J}^{e}\right)$. They are endogenously determined, and will be discussed later on.

There are two intervals of equal length of workers and firms, $\Omega_{I}=\Omega_{J} \equiv(0,1)$, both endowed with the Lebesgue measure. Each interval is partitioned into two sets, $\left\{\Omega_{I}^{n e}, \Omega_{I}^{e}\right\} \equiv \Omega_{I}^{P}$ and $\left\{\Omega_{J}^{n e}, \Omega_{J}^{e}\right\} \equiv \Omega_{J}^{P}$, determined endogenously. Let $\nu\left(\Omega_{I}^{s}\right)$ $\left(\nu\left(\Omega_{J}^{s}\right)\right)$ denote the measure of the set $\Omega_{I}^{s}\left(\Omega_{J}^{s}\right.$, respectively). In sector $s$, production requires a firm $j$ (with physical capital $k_{j}^{s}$ ) and a worker $i$ (with stock of human capital $h_{i}^{s}$ ). Once the partitions $\Omega_{I}^{P}$ and $\Omega_{I}^{P}$ are given, each sector of the economy reduces to the set-up studied in Acemoglu (1996). Firms are identical, and choose their investments in physical capital to maximize their expected profits. Workers choose their investments in human capital to maximize their expected utilities.

The economy lasts one period, divided into several subperiods. We consider two versions of the basic model. We adopt as a benchmark a frictionless (or Walrasian) economy, where, in subperiod 0, firms and workers enter, paying a fixed cost, one of the two sectors. Then, at 1 , each firm active in sector $s$ is matched with a worker active in the same sector (we will be more precise on the matching issue later on). Matched firm and worker sign a binding contract on the amount of human and physical capital that they will supply in subperiod 2. In the final subperiod, investments are carried out, exchanges and production take place, and agents are paid on the basis of their marginal product.

In the second version of the model, the one with frictions, the total output of each match is split according to the Nash bargaining solution with exogenous 
weights $\beta$ and $(1-\beta) .{ }^{8}$ Moreover, and most important, agents cannot contract with their partners a given level of investment, because these are carried out before matches take place. To summarize: in subperiod 0, agents choose to enter one of the two sectors, paying a fixed entry cost. In subperiod 1, they choose their levels of investment. In subperiod 2, they are randomly matched and then, finally, production and exchanges take place.

Technologies are described by a pair of Cobb-Douglas production functions with constant returns to scale. Therefore, in the Walrasian set-up, equilibrium profits are zero, entry costs $d_{j}$ must be zero for each $s$, and each firm is indifferent among sectors. Thus, the equilibrium partition is essentially determined by the labor supply side of the model. On the contrary, in the economy with frictions, expected producers' surpluses are positive in both sectors and, as we will show later on, they are, at the equilibrium, always larger in sector $e$. To avoid additional complications not really germane to our main issue, we want to consider an economy with full employment at the equilibrium. This requires that, at the equilibrium, each agent is actually matched with a partner. We assume, as implicit in Acemoglu (1996), that the matching function guarantees with probability one a match to each agent, provided that $\nu\left(\Omega_{I}^{s}\right)=\nu\left(\Omega_{J}^{s}\right){ }^{9}$ Given the focus of the paper, the partition $\Omega_{I}^{P}$ must be determined endogenously. Hence, to guarantee full employment, we need that, at each equilibrium, $\nu\left(\Omega_{I}^{s}\right)=\nu\left(\Omega_{J}^{s}\right)$. The easiest way to obtain this property is to introduce a feature of the economy such that equilibrium expected profits are always equal in the two sectors. ${ }^{10}$ One way to do it is to assume that the technology exploited in sector $n e$ is free, while the one adopted in sector $e$ is protected by a patent, owned by some outside agent. ${ }^{11}$ Rights to use the patent are auctioned off to firms before the match firm-worker obtains. Given that, at an equilibrium, expected profits in both sectors must be identical, the equilibrium royalties must be equal to the (positive) difference between the expected producer's surpluses in the two sectors. Then, at each equilibrium, each firm is indifferent among sectors, so that we can choose $\Omega_{J}^{P}$ with $\nu\left(\Omega_{I}^{s}\right)=\nu\left(\Omega_{J}^{s}\right)$. The property we are looking for.

Without any loss of generality, the prices of both kinds of output are set equal to 1 and, therefore, we omit them.

Finally, notice that there are always three additional, trivial, equilibria: the ones where all the workers and the firms are in one of the two sectors, and the one where none is active in any sector. As usual, we ignore them.

\footnotetext{
${ }^{8}$ For a rationalization of this allocation rule in this context, see the Appendix in Acemoglu (1996). We assume that $\beta$ is sector-invariant. Given that it is exogenous, to let it vary across sectors would just introduce more notation without any real additional insight.

${ }^{9} \mathrm{~A}$ commonly used function which delivers this property is $\pi_{j}^{s}=\frac{\min \left\{\nu\left(\Omega_{I}^{s}\right), \nu\left(\Omega_{J}^{s}\right)\right\}}{\nu\left(\Omega_{J}^{s}\right)}$, where $\pi_{j}^{s}$ is the probability of a match for a firm active in sector $s$.

${ }^{10} \mathrm{An}$ alternative solution is to assume that firms cannot move across sectors. A non-null measure of firms is exogenously assigned to each sector. We then pick a matching function which always guarantees that each firm is matched with a worker (and conversely) for each non-trivial partition of the workers. As long as there is a continuum of agents in each sector, this can be done. Of course, this approach would break down if we had a finite number of agents and, anyhow, is based on a very ad hoc trick.

${ }^{11}$ Clearly, nothing would change if each technology was subject to a distinct patent. Also: any input used only in sector $e$ and with perfectly inelastic supply would do. We consider the case of a patent to simplify as much as possible the model.
} 


\section{THE FRICTIONLESS ECONOMY}

When active in sector $s$, and matched with worker $i$ with human capital $h_{i}^{s}$, firm $j$ has production function

$$
y_{i j}^{s}=A^{s} h_{i}^{s \alpha} k_{j}^{s(1-\alpha)},
$$

with $A^{e}>A^{n e}$. Let $\mu$ be the unit price of physical capital, that we assume to be equal in the two sectors. This implies some loss of generality, but it simplifies notation and computations. Most important, similar results hold for $\mu^{e} \neq \mu^{n e}$.

If active in sector $s$, and given a match with worker $i$, firm $j$ solves optimization problem

$$
\text { choose } k_{j}^{s} \in \arg \max A^{s} h_{i}^{s \alpha} k_{j}^{s(1-\alpha)}-\mu k_{j}^{s}-w_{i j}^{s} h_{i}^{s} .
$$

For each worker active in sector $s$, the utility function is

$$
U_{i}^{s}\left(C_{i}^{s}, h_{i}^{s}\right)=C_{i}^{s}-\frac{1}{\delta_{i}} \frac{h_{i}^{s(1+\Gamma)}}{1+\Gamma}
$$

where $C_{i}^{s}$ denotes consumption, $h_{i}^{s}$ is the amount of human capital (or the labor supply). Let $c_{I}^{s}$ be the (fixed) cost of the investment in sector $s$ human capital. Then, in the absence of taxes and subsidies, if worker $i$ is active in sector $s$ and matched with firm $j, C_{i}^{s}=\left(w_{i j}^{s} h_{i}^{s}-c_{I}^{s}\right)$. Workers are heterogeneous because of the parameter $\delta_{i}$, indexing their marginal disutility of effort. Without any essential loss of generality, we assume that $\delta_{i}=i$, and that $\delta_{i}$ is uniformly distributed on $(0,1)$. More general assumptions on the distribution of $\delta_{i}$, or its support, would not change any essential result. Given that, in the sequel, we will introduce uniform lumpsum taxes, we must either allow for negative consumption, or assume that workers have a strictly positive, and sufficiently large, initial endowment of consumption good. Given the properties of the utility functions, purely notation-wise, the most convenient solution is the first one.

It is straightforward to check that the amount of agent $i$ 's investment in human capital in sector $s$ is given by $H^{W s}\left(\delta_{i}\right) \equiv\left[\delta_{i} \alpha A^{s \frac{1}{\alpha}}\left(\frac{1-\alpha}{\mu}\right)^{\frac{1-\alpha}{\alpha}}\right]^{\frac{1}{\Gamma}}$, where the superscript $W$ denotes the frictionless economy. Assuming (with no loss of generality because, at the equilibrium, profits are always zero) that firm $j$ is always matched with worker $i=j$, at the equilibrium, we can write the demand for physical capital of firm $j=i$ as $K^{W s}\left(\delta_{i}\right) \equiv\left[\delta_{i} \alpha A^{s \frac{1+\Gamma}{\alpha}}\left(\frac{1-\alpha}{\mu}\right)^{\frac{1+\Gamma-\alpha}{\alpha}}\right]^{\frac{1}{\Gamma}}$.

Let's now consider the equilibrium partition $\Omega_{I}^{P}$. For convenience (here and in the sequel), set $c_{I}^{n e}=0$ and $c_{I}^{e}>0$. Let $V^{W s}\left(\delta_{i}\right) \equiv U_{i}^{s}\left(H^{W s}\left(\delta_{i}\right), K^{W s}\left(\delta_{i}\right)\right)$, be the level of utility of agent $i$ active in sector $s$, evaluated at the equilibrium. Worker $i$ chooses to enter sector $e$ if and only if $V^{W e}\left(\delta_{i}\right)-V^{W n e}\left(\delta_{i}\right) \geq 0$. A straightforward computation shows that this inequality is satisfied if and only if

$$
\delta_{i} \geq \delta^{W} \equiv\left[\frac{1+\Gamma}{\Gamma} c_{I}^{e}\right]^{\Gamma}\left[\left(\alpha\left(\frac{1-\alpha}{\mu}\right)^{\frac{1-\alpha}{\alpha}}\right)^{\frac{1+\Gamma}{\Gamma}}\left(A^{e \frac{1+\Gamma}{\alpha \Gamma}}-A^{n e \frac{1+\Gamma}{\alpha \Gamma}}\right)\right]^{-\Gamma} .
$$

Hence, for $c_{I}^{e}$ positive and sufficiently small, there is a unique threshold value $\delta^{W}$, strictly increasing in $c_{I}^{e}$. All the agents with $\delta_{i}<\delta^{W}$ do not invest in education, 
while the ones with $\delta_{i} \geq \delta^{W}$ do. ${ }^{12}$

\section{THE ECONOMY WITH FRICTIONS: EQUILIBRIA AND THEIR COMPARATIVE STATICS PROPERTIES}

Later on, we will show that, at the equilibrium, it is always $\Omega_{I}^{e}=\left[\delta^{F}, 1\right)$, where $\delta^{F}$ denote the equilibrium value of the threshold in the economy with frictions. Hence, we can restrict the analysis to partitions $\Omega_{I}^{P}$ and $\Omega_{J}^{P}$ defined by an arbitrary level of the threshold, denoted $\widehat{\delta}$. To emphasize this, we use the notation $\Omega_{J}^{s}(\widehat{\delta})$ and $\Omega_{I}^{s}(\widehat{\delta})$.

For future reference, let's determine the optimal amount of investments assuming that there is a public intervention defined by a pair of vectors $\xi^{s} \equiv\left(\tau^{s}, \zeta^{s}, \Delta c_{I}^{s}, T\right)$, $\xi \equiv\left(\xi^{e}, \xi^{n e}\right)$, describing (possibly) sector specific subsidies and taxes. We assume that there are linear subsidies on labor income (with rates $\tau^{s}, s=n e, e$ ), and on the cost of the investments in physical capital (with rates $\zeta^{s}, s=n e, e$ ), and fixed taxes on the direct costs of education, $\Delta c_{I}^{s}$ (we will always set $\Delta c_{I}^{n e}=0$ ). $T$ denotes a (uniform) lump-sum tax, such that the public budget is balanced. We write the subsidy rates as sector specific just to simplify the notation. At equilibrium, this system of subsidies is isomorphic to a system of step-linear subsidies to labor income and to investments in physical capital. ${ }^{13}$ Also, bear in mind that our main qualitative results hold if we start with an arbitrary (linear) tax system, and interpret $\left(\tau^{e}, \tau^{n e}\right)$ as small changes in the tax rates applied to the two classes of workers. Then, $\tau^{e} \leq 0$ and $\tau^{n e} \geq 0$ would define a move from a flat labor income tax to a progressive one.

Pick an arbitrary threshold $\widehat{\delta}$. If active in sector $s$, firm $j$ selects the value of $k_{j}^{s}$ solving the expected profits maximization problem

$$
\begin{aligned}
\operatorname{choose} k_{j}^{s} & \in \arg \max _{k_{j}^{s}} E_{\Omega_{I}^{s}(\widehat{\delta})}\left((1-\beta) A^{s} h_{i}^{s \alpha} k_{j}^{s(1-\alpha)}-\mu\left(1-\zeta^{s}\right) k_{j}^{s}\right)-d_{J}^{s} \\
& =(1-\beta) A^{s} E_{\Omega_{I}^{s}(\widehat{\delta})}\left(h_{i}^{s \alpha}\right) k_{j}^{s(1-\alpha)}-\mu\left(1-\zeta^{s}\right) k_{j}^{s}-d_{J}^{s},
\end{aligned}
$$

where, given any random variable $x^{s}$, with $x^{s}: \Omega_{I}^{s} \rightarrow \mathbb{R}$, (or $y^{s}$, with $y^{s}: \Omega_{J}^{s} \rightarrow \mathbb{R}$ ), $E_{\Omega_{I}^{s}(\widehat{\delta})}\left(x_{j}^{s}\right) \equiv \frac{\int_{\Omega_{I}^{s}(\widehat{\delta})}^{s} x_{i}^{s} d i}{\nu\left(\Omega_{I}^{s}(\widehat{\delta})\right)}\left(\right.$ or $\left.E_{\Omega_{J}^{s}(\widehat{\delta})}\left(y_{i}^{s}\right)\right)$ denotes the conditional expectation of $x_{i}^{s}$ over the set $\Omega_{I}^{s}(\widehat{\delta})$ (or of $y_{j}^{s}$ over $\Omega_{J}^{s}(\widehat{\delta})$ ).

The pair of maps $K^{s}\left(\widehat{\delta}, E_{\Omega_{I}^{s}(\widehat{\delta})}\left(h_{i}^{s \alpha}\right), \xi\right), s=n e, e$, defines the optimal investment in physical capital for the firms active in the two sectors. They are $j$-invariant because firms in each sector are identical, and depend upon the exogenous vector $\xi$, the arbitrary threshold $\widehat{\delta}$, and the conditional expectations $E_{\Omega_{I}^{s}(\widehat{\delta})}\left(h_{i}^{s \alpha}\right)$. Let $\Pi^{s}\left(\delta_{i}, \widehat{\delta}, E_{\Omega_{I}^{s}(\widehat{\delta})}\left(h_{i}^{s \alpha}\right), \xi\right)$ be the surplus (because inclusive of $\left.d_{J}^{s}\right)$ of the firm matched with worker $i$ in sector $s$.

\footnotetext{
${ }^{12}$ Evidently, the agent with $\delta_{i}=\delta^{W}$ is indifferent between the two choices. We assume that any indifferent agent will actually choose to invest.

${ }^{13}$ Exactly the same closed form of the equilibrim is obtained considering a direct subsidy to effort in education of the form $\tau^{s} h_{i}^{s \alpha}$, which, however, would require direct observability of effort.
} 
The optimization problem of worker $i$ (if active in $s$ ) is

$$
\begin{aligned}
& \text { choose } h_{i}^{s} \in \arg \max _{h_{i}^{s}} E_{\Omega_{J}^{s}(\widehat{\delta})}\left(U_{i}^{s}(.)\right) \\
\equiv & \left(1+\tau^{s}\right) \beta A^{s} h_{i}^{s \alpha} E_{\Omega_{J}^{s}(\widehat{\delta})}\left(k_{j}^{s(1-\alpha)}\right)-\frac{1}{\delta_{i}} \frac{h_{i}^{s(1+\Gamma)}}{1+\Gamma}-\left(c_{I}^{s}+\Delta c_{I}^{s}+T\right) .
\end{aligned}
$$

The pair of maps $H^{s}\left(\delta_{i}, \widehat{\delta}, E_{\Omega_{J}^{s}(\widehat{\delta})}\left(k_{j}^{s(1-\alpha)}\right), \xi\right), s=n e, e$, describes the optimal investments in human capital of the agents in each sector, parameterized by the variable $\delta_{i}$.

Let $V^{s}\left(\delta_{i}, \widehat{\delta}, E_{\Omega_{J}^{s}(\widehat{\delta})}\left(k_{j}^{s(1-\alpha)}\right), \xi\right)$ be the associated level of utility of agent $i$, if active in sector $s$. Worker $i$ enters sector $e$ if and only if

$$
\begin{aligned}
& F\left(\delta_{i}, \widehat{\delta}, E_{\Omega_{J}^{s}(\widehat{\delta})}\left(k_{j}^{s(1-\alpha)}\right), \xi\right) \\
\equiv & V^{e}\left(\delta_{i}, \widehat{\delta}, E_{\Omega_{J}^{e}(\widehat{\delta})}\left(k_{j}^{e(1-\alpha)}\right), \xi\right)-V^{n e}\left(\delta_{i}, \widehat{\delta}, E_{\Omega_{J}^{n e}(\widehat{\delta})}\left(k_{j}^{n e(1-\alpha)}\right), \xi\right) \geq 0
\end{aligned}
$$

where $F\left(\delta_{i}, \widehat{\delta}, E_{\Omega_{J}^{s}(\widehat{\delta})}\left(k_{j}^{s(1-\alpha)}\right), \xi\right)$ is agent $i$ 's utility gain due to his investment in education.

It is easy to check that, for each given $\left(\widehat{\delta}, E_{\Omega_{J}^{s}(\widehat{\delta})}\left(k_{j}^{s(1-\alpha)}\right), \xi\right), F($.$) is strictly$ increasing in $\delta_{i}$.

Let's now make precise our notion of equilibrium.

Definition 1. Given $\xi$, an equilibrium of the economy with frictions is a threshold value $\delta^{F} \in(0,1)$, and a royalty $d_{J}^{e F}>0$, such that:

i. $\quad K^{s}\left(\delta^{F}, E_{\Omega_{I}^{s}\left(\delta^{F}\right)}\left(H^{s}(.)^{\alpha}\right), \xi\right)$ solves $\left(\Pi^{s}\right), s=$ ne for each $j=i$ such that $\delta_{i}<\delta^{F}, s=e$ for each $j=i$ such that $\delta_{i} \geq \delta^{F}$;

ii. $\quad H^{s}\left(\delta_{i}, \delta^{F}, E_{\Omega_{J}^{s}\left(\delta^{F}\right)}\left(K^{s}(.)^{(1-\alpha)}\right), \xi\right)$ solves $\left(U^{s}\right), s=$ ne for $\delta_{i}<\delta^{F}$, and $s=e$ for $\delta_{i} \geq \delta^{F}$;

iii. $\quad E_{\Omega_{I}^{e}\left(\delta^{F}\right)}\left(\Pi^{e}\left(\delta_{i}, \delta^{F}, E_{\Omega_{I}^{e}\left(\delta^{F}\right)}\left(H^{e}(.)^{\alpha}\right), \xi\right)\right)-$

$E_{\Omega_{I}^{n e}\left(\delta^{F}\right)}\left(\Pi^{n e}\left(\delta_{i}, \delta^{F}, E_{\Omega_{I}^{n e}\left(\delta^{F}\right)}\left(H^{n e}(.)^{\alpha}\right), \xi\right)\right)=d_{J}^{e F}>0$

iv. $\quad F\left(\delta_{i}, \delta^{F}, E_{\Omega_{J}^{s}\left(\delta^{F}\right)} K^{s}(.)^{(1-\alpha)}, \xi\right) \geq 0$ if and only if $\delta_{i} \geq \delta^{F}$.

First, observe that the conditional expectations $\left\{E_{\Omega_{J}^{s}\left(\delta^{F}\right)}\left(k_{j}^{s(1-\alpha)}\right), E_{\Omega_{I}^{s}\left(\delta^{F}\right)}\left(h_{i}^{s \alpha}\right)\right\}$, $s=n e, e$, are computed making reference to the actual values $\left\{H^{s}(),. K^{s}().\right\}$, so that we are imposing rational expectations. Conditions $(i-i i)$ impose individual optimality in the choice of the investment. Conditions $(i i i-i v)$ impose individual optimality in the choice of the sector where an agent is active. Notice that iii implies that each firm is indifferent between being active in any of the two sectors, so that we can impose $\Omega_{J}^{P}=\Omega_{I}^{P}=\left\{\left(0, \delta^{F}\right),\left[\delta^{F}, 1\right)\right\}$ (by $\left.i v\right)$.

The main results concerning existence of equilibria and their properties are summarized in Prop. 1. The proof is in Appendix 1. Here we just provide an outline of the argument: first, given an arbitrary $\widehat{\delta}$, we compute the values of $\left\{\widetilde{H}^{s}\left(\delta_{i}, \widehat{\delta}, \xi\right), \widetilde{K}^{s}(\widehat{\delta}, \xi)\right\}, s=n e, e$, the demand functions for investments in human and physical capital obtained imposing that (conditional on $\widehat{\delta}$ ) expectations are fulfilled. Occasionally, we will refer to $\left(\widetilde{H}^{s}\left(\delta_{i}, \widehat{\delta}, \xi\right), \widetilde{K}^{s}(\widehat{\delta}, \xi)\right)$, and the derived maps $\widetilde{V}^{s}\left(\delta_{i}, \widehat{\delta}, \xi\right)$ and $\widetilde{\Pi}^{s}\left(\delta_{i}, \widehat{\delta}, \xi\right)$, as the equilibrium maps conditional on $\widehat{\delta}$. 
Let $\widetilde{F}\left(\delta_{i}, \widehat{\delta}, \xi\right)$ be the analogous of map $F($.$) , obtained using \left\{\widetilde{H}^{s}\left(\delta_{i}, \widehat{\delta}, \xi\right), \widetilde{K}^{s}(\widehat{\delta}, \xi)\right\}$. If $\widetilde{F}\left(\delta_{i}, \widehat{\delta}, \xi\right)$ is strictly increasing in $\delta_{i}, \widetilde{F}\left(\delta_{i}, \widehat{\delta}, \xi\right)=0$ at $\delta_{i}=\widehat{\delta}$ gives us the equilibrium value of the threshold, i.e., $\delta^{F}$. Hence, $\delta^{F}$ is the solution to the equation

$$
\widetilde{F}\left(\delta_{i}=\widehat{\delta}, \widehat{\delta}, \xi\right) \equiv f(\widehat{\delta}, \xi)-a\left(c_{I}^{e}+\Delta c_{I}^{e}\right)=0,
$$

where, by direct computation (and using eqs. (A3) and (A4)),

$$
\begin{aligned}
f(\widehat{\delta}, \xi) \equiv & \widehat{\delta}^{\frac{\alpha}{1+\Gamma-\alpha}}\left(A^{e} E_{\Omega_{I}^{e}(\widehat{\delta})}\left(\delta_{i}^{\frac{\alpha}{1+\Gamma-\alpha}}\right)^{(1-\alpha)}\right)^{\frac{1+\Gamma}{\alpha \Gamma}} \chi^{e}(\xi)- \\
& \widehat{\delta}^{\frac{\alpha}{1+\Gamma-\alpha}}\left(A^{n e} E_{\Omega_{I}^{n e}(\widehat{\delta})}\left(\delta_{i}^{\frac{\alpha}{1+\Gamma-\alpha}}\right)^{(1-\alpha)}\right)^{\frac{1+\Gamma}{\alpha \Gamma}} \chi^{n e}(\xi),
\end{aligned}
$$

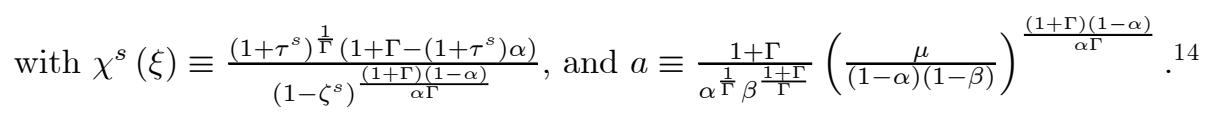

Proposition 1. Fix $(\Gamma, \alpha, \beta)$. Given $\left(A^{e}, A^{\text {ne }}, \xi=0\right)$, there is $\widetilde{C}>0$ such that, for each $c_{I}^{e}$ with ac $c_{I}^{e} \in(0, \widetilde{C})$, there is an equilibrium with threshold value $\delta^{F} \in$ $(0,1)$. Moreover, given $A^{\text {ne }}$, there is $\underline{A}^{e}$ such that, for each $A^{e}>\underline{A}^{e}$, at $\xi=0$, the equilibrium is unique and $\left.\frac{\partial f(.)}{\partial \hat{\delta}}\right|_{\widehat{\delta}=\delta^{F}}>0$. Also, $\frac{\partial \delta^{F}(.)}{\partial \tau^{e}}<0, \frac{\partial \delta^{F}(.)}{\partial \tau^{n e}}>0, \frac{\partial \delta^{F}(.)}{\partial \Delta c_{I}^{e}}>0$, $\frac{\partial \delta^{F}(.)}{\partial A^{e}}<0$ and $\frac{\partial \delta^{F}(.)}{\partial A^{n e}}>0$, where $\delta^{F}\left(A^{e}, A^{n e}, \xi\right)$ is the function associating with the vector $\left(A^{e}, A^{\text {ne }}, \xi\right)$ the (unique) equilibrium threshold.

Proof. See Appendix 1.

In the sequel, we will mostly consider the leading case where $\left.\frac{\partial f(.)}{\partial \hat{\delta}}\right|_{\widehat{\delta}=\delta^{F}}>0$ at each equilibrium threshold, i.e., we restrict the analysis to economies where $\frac{A^{e}}{A^{n e}}$ is sufficiently large. Also, with some abuse of notation, we will use $\delta^{F}(\xi)$ to denote the equilibrium threshold as a function of $\xi$ whenever $\left(A^{e}, A^{n e}\right)$ are treated as fixed.

Remark 1. The restriction to economies with $\left.\frac{\partial f(.)}{\partial \hat{\delta}}\right|_{\widehat{\delta}=\delta^{F}}>0$ at each $\delta^{F}$ delivers two different properties of equilibria. It is easy to check that, for $\widehat{\delta}$ sufficiently close to 0 , we always have $\frac{\partial f(.)}{\partial \hat{\delta}}>0$. Hence, $\left.\frac{\partial f(.)}{\partial \hat{\delta}}\right|_{\widehat{\delta}=\delta^{F}}>0$ at each $\delta^{F}$ implies uniqueness of equilibria. Moreover, by the implicit function theorem, the comparative statics effects $\left(\frac{\partial \delta^{F}(.)}{\partial \tau^{s}}, \frac{\partial \delta^{F}(.)}{\partial \Delta c_{I}^{e}}, \frac{\partial \delta^{F}(.)}{\partial A^{s}}\right)$ depend upon the the derivatives of the equilibrium conditions with respect to $\left(A^{e}, A^{n e}, \xi\right)$ and $\widehat{\delta}$. It turns out (see the proof of Prop. 1) that the signs of the derivatives with respect to $\left(A^{e}, A^{n e}, \xi\right)$ are always uniquely defined. It follows that the signs of the comparative statics effects just depend upon the sign of $\left.\frac{\partial f(.)}{\partial \widehat{\delta}}\right|_{\widehat{\delta}=\delta^{F}}$. Hence, to restrict the analysis to economies such that $\left.\frac{\partial f(.)}{\partial \hat{\delta}}\right|_{\widehat{\delta}=\delta^{F}}>0$ at each $\delta^{F}$ allows us to obtain well-defined comparative statics results. Example A1 in Appendix exhibits an economy with $\frac{\partial f(.)}{\partial \widehat{\delta}}<0$ for $\widehat{\delta}$ sufficiently close to 1 . Given that $\frac{\partial f(.)}{\partial \hat{\delta}}$ is a continuous function on $(0,1)$, for this economy $f($.$) has at least one local maximum, \bar{\delta}$. Evidently, each economy with $c_{I}^{e}$ such that $a c_{I}^{e}<f(\bar{\delta})$, and close enough to $f(\bar{\delta})$, has at least two equilibria. Hence,

\footnotetext{
${ }^{14}$ To avoid misunderstandings: $\widetilde{F}\left(\delta_{i}, \widehat{\delta}, \xi\right)$, strictly increasing in $\delta_{i}$. The function $f(\widehat{\delta}, \xi)$ is obtained setting $\delta_{i}=\widehat{\delta}$ and it does not necessarily have this property.
} 
this example shows that some additional restrictions on the parameters (such as the ones provided in Prop. 1) are required to obtain uniqueness, and well-defined comparative statics properties, of equilibria.

Remark 2. Let $f\left(\widehat{\delta}, A^{e}, A^{n e}\right)$ be the function obtained from $f(\widehat{\delta}, \xi)$ setting $\xi=0$ and making explicit its dependence on $\left(A^{e}, A^{n e}\right)$ (similarly for $\delta^{F}\left(A^{e}, A^{n e}\right)$ ). It is easy to check that

$\lim _{\widehat{\delta} \rightarrow 1} f\left(\widehat{\delta}, A^{e}, A^{n e}\right)=\left(A^{e \frac{1+\Gamma}{\alpha \Gamma}}-\left(\frac{1+\Gamma-\alpha}{1+\Gamma}\right)^{\frac{(1-\alpha)(1+\Gamma)}{\alpha \Gamma}} A^{n e \frac{1+\Gamma}{\alpha \Gamma}}\right)(1+\Gamma-\alpha)>0$.

Consider a sequence of equilibrium thresholds $\left\{\delta^{F}\left(A^{e v}, A^{n e}\right)\right\}_{v=1}^{v=\infty}$ associated with any sequence $\left\{A^{e v}\right\}_{v=1}^{v=\infty}$ with $A^{e v} \rightarrow A^{\text {ne }}$. Given that the limit computed above is positive at $A^{e}=A^{n e}$, there is an interval of values of $c_{I}^{e}$ such that the associated equilibrium threshold is strictly smaller than $1 .{ }^{15}$ Hence, investments in human capital of type $e$ may be positive, at the equilibrium, even when this skill is completely useless, from the technological viewpoint. Indeed, when $A^{e}=A^{\text {ne }}$, the two sectors are essentially identical, while to operate in sector $e$ requires the use of costlier skills. Clearly, Pareto efficiency would require us to shut down sector $e$. This is similar to what happens in signalling models. The mechanism behind positive investments in technically useless skills is crucially related to the lack of contractibility and to the workers' self-selection into the two sectors. It is an open issue how general this asymptotic property of the generalized Roy model is in non-Walrasian economies.

Remark 3. Fix $\xi=0$. Modulo a redistribution of output, the Walrasian allocation is the unique Pareto efficient allocation of this economy (i.e., $\delta^{W}$ coincides with its Pareto optimal level). With elastic supply of human and physical capital, no allocation rule (i.e., no value of $\beta$ ) can guarantee Pareto efficiency of the equilibrium allocation, because, in the economy with frictions, a firm's investment in physical capital does not depend upon the value of $\delta_{i}$ of the worker it is matched with, while it does in the Walrasian economy.

Remark 4. At $\xi=0$, using $(A 3)$ and $(A 4)$ in Appendix 1, and by a straightforward computation, the physical/human capital ratio is given by

$$
\frac{\widetilde{K}^{s}\left(\delta^{F}\right)}{\widetilde{H}^{s}\left(\delta_{i}, \delta^{F}\right)}=\left[\frac{(1-\beta)^{\frac{1}{\alpha}} E_{\Omega_{I}^{s}\left(\delta^{F}\right)}\left(\delta_{i}^{\frac{a}{1+\Gamma-\alpha}}\right)^{\frac{1}{\alpha}}}{\delta_{i}^{1+\Gamma-\alpha}}\right] \frac{K^{W s}\left(\delta_{i}\right)}{H^{W s}\left(\delta_{i}\right)} .
$$

In sector $n e$, and for sufficiently small $\delta_{i}$, the term in square brackets is always greater than one, so that $\frac{\widetilde{K}^{n e}\left(\delta^{F}\right)}{\widetilde{H}^{n e}\left(\delta_{i}, \delta^{F}\right)}>\frac{K^{W n e}\left(\delta_{i}\right)}{H^{W n e}\left(\delta_{i}\right)}$, for $\delta_{i}$ small enough. This immediately implies that agents with a sufficiently low $\delta_{i}$ are always better off at the equilibrium of the frictional economy. Hence, the Walrasian equilibrium allocation is not Pareto superior to the one of the economy with frictions. Of course, it still dominates it in terms of total surplus.

Remark 5. The threshold value $\delta^{F}$ can be either lower or higher than its value in the Walrasian economy. For instance, let $\xi=0$, and set $A^{e}=2, A^{\text {ne }}=1$, $\alpha=\beta=1 / 2$, and $\Gamma=1$. By direct computation, one can verify that, for $c_{I}^{e}<0.019$,

\footnotetext{
${ }^{15}$ Depending upon the values of the other parameters, we may have (at least) two equilibria with different thresholds, or a unique equilibrium.
} 
$\delta^{F}<\delta^{W}$, while, for $c_{I}^{e}>0.019$, the opposite occurs. Hence, lack of contractibility always induces Pareto inefficiency because of lower than optimal investments, while it has an ambiguous effect on the size of the set of people investing in education. From this viewpoint, therefore, it does not induces unambiguously overeducation (or undereducation).

Our main purpose is to analyze the policy implications of workers' self-selection into distinct labor markets. However, it is interesting to consider the comparative statics of equilibria, also because, obviously, the welfare effects of different policies comes through their impact on the equilibrium values of the endogenous variables.

Let $\phi \equiv\left(A^{e}, A^{n e}, \xi\right)$. Let $w^{s}\left(\delta_{i}, \delta^{F}(\phi), \phi\right)$ be worker $i$ 's wage in sector $s$. The standard deviation, $\sigma_{\Omega_{I}^{s}\left(\delta^{F}\right)}\left(\delta^{F}(\phi), \phi\right)$, measures the variability of wages within sector $s . W P_{\Omega_{I}^{e}\left(\delta^{F}\right)}\left(\delta^{F}(\phi), \phi\right)$ is the wage premium. ${ }^{16}$ With tedious, but straightforward (therefore, omitted), computations, we obtain the following comparative statics results.

Proposition 2. Fix $\left(\Gamma, c_{I}^{e}, \alpha, \beta\right)$. Assume that $\left.\frac{\partial f(.)}{\partial \widehat{\delta}}\right|_{\widehat{\delta}=\delta^{F}(\phi)}>0$. At $\xi=0$, the following sign restrictions are satisfied: ${ }^{17}$

$$
\left[\begin{array}{cccccc} 
& d \tau^{e} & d \tau^{n e} & \Delta c_{I}^{e} & d A^{e} & d A^{n e} \\
E_{\Omega_{I}^{e}\left(\delta^{F}\right)}\left(\widetilde{H}^{e}(.)\right) & ? & + & + & ? & + \\
E_{\Omega_{I}^{n e}\left(\delta^{F}\right)}\left(\widetilde{H}^{n e}(.)\right) & - & + & + & - & + \\
\widetilde{K}^{e}(.) & ? & + & + & ? & + \\
\widetilde{K}^{n e}(.) & - & + & + & - & + \\
E_{\Omega_{I}^{e}\left(\delta^{F}\right)}\left(w^{e}(.)\right) & ? & + & + & ? & + \\
E_{\Omega_{I}^{n e}\left(\delta^{F}\right)}\left(w^{n e}(.)\right) & - & + & + & - & + \\
\sigma_{\Omega_{I}^{n e}\left(\delta^{F}\right)}(.) & - & + & + & - & + \\
W P_{\Omega_{I}^{e}\left(\delta^{F}\right)}(.) & + & - & - & + & -
\end{array}\right] .
$$

The intuition behind these results is based on the interaction of the incentive and the composition effect. For instance, consider a change in the parameter $\tau^{n e}$ (an increase in the marginal subsidy - or, if we take as a benchmark an economy with a flat labor income tax, a reduction in the marginal tax rate - on the income of the $n e$ workers). As a pure incentive effect, its increase stimulates effort in education of low-skilled workers, and pushes up the threshold $\delta^{F}(\phi)$. Then, through the composition effect, it improves the (conditional) expected human capital of both low and high skilled workers. This, in turn, stimulates investments in physical capital in both sectors. The positive feed-backs strengthen the initial impacts. Hence, the effects on expected human and physical capitals and on wages are positive in both sectors. For the wage premium, by direct computation, it turns out that both direct

\footnotetext{
${ }^{16}$ In general, there are three different notions of wage premium: $\frac{w^{e}(.)}{w^{n e}(.)}$ at $\delta_{i}=\delta^{F}(\phi)$, $E_{\Omega_{I}^{e}\left(\delta^{F}\right)}\left(\frac{w^{e}(.)}{w^{n e}(.)}\right)$ and $E_{\Omega_{I}^{n e}\left(\delta^{F}\right)}\left(\frac{w^{e}(.)}{w^{n e}(.)}\right)$. Due to linearity in $\delta_{i}$ of the wage function, here they coincide.

${ }^{17}$ Each cell reports the sign of the derivative of the function on the row with respect to the variable on the column. We omit the standard deviation of the wages of skilled workers. For this variable, is impossible to reach any well-defined, general result. For reasonable values of the parameters, $\alpha=\frac{2}{3}$ and $\Gamma>\frac{1}{2}$, some numerical simulations show that the composition effect has the sign opposite to the one of $\frac{\partial \delta^{F}}{\partial \phi}$. Therefore, $\frac{\partial \sigma_{\Omega_{I}^{e}(\delta F)}(\cdot)}{\partial \phi}$ is positive for $\phi^{\prime} \in\left\{\tau^{e}, A^{e}\right\}$, negative for $\phi^{\prime} \in\left\{\tau^{n e}, \Delta c_{I}^{e}, A^{n e}\right\}$.
} 
and composition effects are negative. The standard deviation of wages of unskilled workers increases because both effects are positive. On the other hand, an increase in the value of $\tau^{e}$ has unambiguously a negative effect on the level of human (and, consequently, of physical) capital and on the wages in the ne sector, because it decreases the value of $\delta^{F}(\phi)$ (composition effect). Indeed, given that the expected human capital of the pool of ne workers decreases because of the increase in $\tau^{e}$, physical capital also decreases, depressing further the workers' optimal investments in this sector. The impact of $d \tau^{e}>0$ in the $e$ sector is ambiguous because the incentive effect stimulates additional investment in human capital. However, the composition effect acts in the opposite direction, because $d \tau^{e}>0$ induces workers with a (relatively) low value of $\delta_{i}$ to switch sector, with a negative impact on the expected level of human capital in the $e$ sector and, therefore, on the investments in physical capital, inducing a negative feed-back. The effect of exogenous changes in technology, $\left(d A^{e}, d A^{n e}\right)$, can be explained exactly in the same terms. In particular, notice that, in this set-up, "skill biased" technical change $\left(d A^{e}>0, d A^{n e}=0\right)$ has a negative impact on the expected human, and physical, capital and on the wages in the "low skill" sector, an ambiguous impact in the "high skill" one.

\section{EFFICIENCY PROPERTIES OF EQUILIBRIA}

In Remark 3, we have argued that the equilibria of the economy with frictions are Pareto inefficient. We will now show that they do not satisfy either a weaker criterion of constrained optimality ( $\mathrm{CO}$ in the sequel) which takes into account the imperfections which characterize the economy. Most interesting it is the analysis of their inefficiency in terms of the amount, and type, of investments. In the sequel, we will mainly refer to investments in human capital. Similar considerations hold for the ones in physical capital.

In our set-up, inefficiencies can be of two different types. First, an individual can choose an amount of investment different from the CO one, given the partition $\Omega_{I}^{P}$ associated with the $\mathrm{CO}$ allocation. We will refer to this possible source of inefficiency as underinvestment (or overinvestment) in educational effort. Secondly, an agent can choose to invest in a type of education different from the one assigned to her at the CO allocation. We will say that there is underinvestment in educational level when agent $i$ invests in education ne, while, at the $\mathrm{CO}$ allocation, she should invest in education level $e$.

In the one-sector model, equilibria are unambiguously characterized by underinvestment in educational effort. In our set up, the same effect is at work: in each sector, given any arbitrary $\widehat{\delta}$, an increase in the investments of firms and workers leads to a Pareto improvement. Once we consider an arbitrarily given threshold $\widehat{\delta}$, the argument is identical to the one in Acemoglu (1996). Set $\xi=0$ (and omit it, for notational convenience). Fix $\widehat{\delta}$, so that each sector is identical to the economy analyzed there, and consider a small change in the investment of each agent. The changes in utilities and producers' surplus evaluated at the equilibrium (conditional on $\widehat{\delta}$ ) pair $\left(\widetilde{H}^{s}\left(\delta_{i}, \widehat{\delta}\right), \widetilde{K}^{s}(\widehat{\delta})\right)$ (and taking into account that investments in physical capital are $j$-invariant) are given by

$0<\left(\alpha \beta A^{s}\left(\frac{\widetilde{K}^{s}(\widehat{\delta})}{\widetilde{H}^{s}\left(\delta_{i}, \widehat{\delta}\right)}\right)^{1-\alpha}-\frac{1}{\delta_{i}} \widetilde{H}^{s}\left(\delta_{i}, \widehat{\delta}\right)^{\Gamma}\right) d h+\left((1-\alpha) \beta A^{s}\left(\frac{\widetilde{H}^{s}\left(\delta_{i}, \widehat{\delta}\right)}{\widetilde{K}^{s}(\widehat{\delta})}\right)^{\alpha}\right) d k$, 
and

$$
\begin{aligned}
0< & \left((1-\alpha)(1-\beta) A^{s} \frac{E_{\Omega_{I}^{s}(\widehat{\delta})}\left(\widetilde{H}^{s}\left(\delta_{i}, \widehat{\delta}\right)^{\alpha}\right)}{\widetilde{K}^{s}(\widehat{\delta})^{\alpha}}-\mu\right) d k \\
& +\left(\alpha(1-\beta) A^{s} \frac{\widetilde{K}^{s}(\widehat{\delta})^{(1-\alpha)}}{E_{\Omega_{I}^{s}(\widehat{\delta})}\left(\widetilde{H}^{s}\left(\delta_{i}, \widehat{\delta}\right)^{(1-\alpha)}\right)}\right) d h,
\end{aligned}
$$

respectively. The inequalities hold because the first terms in parenthesis in (3) and (4) are zero (at the optimal solutions of $\left(\Pi^{s}\right)$ and $\left(U^{s}\right)$ ), while the second terms are positive. Hence, given any $\widehat{\delta}$, there is underinvestment in educational effort and physical capital, in each sector. This establishes, in a more direct way, the Pareto inefficiency of equilibria in our economy.

In the two-sector case, there is a second potential source of inefficiency, because changes in the value of $\widehat{\delta}$ may also entail Pareto improvements. An increase in the threshold value $\widehat{\delta}$ increases the conditional expected amount of human capital in both sectors at the same time and, consequently, induces an increase in the amount of physical investments of firms in both sectors. Indeed, given that $\delta_{i}^{\frac{\alpha}{1+\Gamma-\alpha}}$ is strictly monotonically increasing,

$$
\frac{\partial E_{\Omega_{I}^{s}(\widehat{\delta})}\left(\delta_{i}^{\frac{\alpha}{1+\Gamma-\alpha}}\right)}{\partial \widehat{\delta}}>0, \text { for each } s \text { and } \widehat{\delta},
$$

and, consequently, using $(A 3)$ and $(A 4)$,

$$
\frac{\partial \widetilde{H}^{s}\left(\delta_{i}, \widehat{\delta}\right)}{\partial \widehat{\delta}}>0 \text { and } \frac{\partial \widetilde{K}^{s}(\widehat{\delta})}{\partial \widehat{\delta}}>0, \text { for each } s \text { and } \widehat{\delta} .
$$

More relevant, from $(A 5),(A 6)$ and (5), for each $i$ and $\widehat{\delta}$,

$$
\frac{\partial \widetilde{V}^{s}\left(\delta_{i}, \widehat{\delta}\right)}{\partial \widehat{\delta}}>0 \quad \text { and } \quad \frac{\partial E_{\Omega_{I}^{s}(\widehat{\delta})}\left(\widetilde{\Pi}^{s}\left(\delta_{i}, \widehat{\delta}\right)\right)}{\partial \widehat{\delta}}>0 .
$$

These properties do not suffice to establish our claim, because a change in the threshold induces a jump in the producer's surplus for the firms shifting from one sector to the other. However, as we will formally establish in Prop. 4, under suitable restrictions, a sufficiently small increase of the value of the threshold increases expected total surplus.

To complete the analysis of the welfare properties of equilibria, it is convenient to introduce an explicit notion of (constrained) efficiency. As usual in economies with frictions, we consider the metaphor of a benevolent planner choosing an allocation while facing constraints aiming to capture the ones the agents face in the decentralized economy. We provide two results. First, we show that there are constrained optimal allocations (Prop. 3), and that they can be attained with an appropriate system of taxes and subsidies (Corollary 1). The amount of subsidies and taxes is entirely dictated by the features of the $\mathrm{CO}$ allocation, and they can be (in fact, they are) quite large. That's why, in Prop. 4 and its Corollary, we study the effects of small taxes and subsidies on total surplus evaluated at the market equilibrium, taking as given the actual demand and supply functions of the agents. Prop. 4 
considers taxes and subsidies balanced with lump-sum taxes. The Corollary taxes on labor income balanced by subsidies to the direct costs of education.

Bear in mind that, in the sequel, we always consider changes in total surplus. We are not concerned with actual Pareto improvements. However, given that utility functions are quasi-linear, an increase in total surplus immediately translates (modulo an appropriate - and $i$-contingent - system of lump-sum taxes and transfers) into a Pareto improvement.

\subsection{Constrained optimal allocations}

The objective function of the planner is defined as the sum of the expected utilities and producers' surpluses of the agents, i.e.,

$$
\begin{aligned}
P\left(h_{i}^{s}, k_{j}^{s}, \Omega_{I}^{s}, \Omega_{J}^{s}\right) \equiv & \sum_{s} \int_{\Omega_{I}^{s}(\widehat{\delta})}\left[\beta E_{\Omega_{J}^{s}(\widehat{\delta})}\left(A^{s} h_{i}^{s \alpha} k_{j}^{s(1-\alpha)}\right)-\frac{1}{\delta_{i}} \frac{h_{i}^{s(1+\Gamma)}}{1+\Gamma}-c_{I}^{s}\right] d i \\
& +\sum_{s} \int_{\Omega_{J}^{s}(\widehat{\delta})}\left[(1-\beta) E_{\Omega_{I}^{s}(\widehat{\delta})}\left(A^{s} h_{i}^{s \alpha} k_{j}^{s(1-\alpha)}\right)-\mu k_{j}^{s}\right] d j .
\end{aligned}
$$

The planner's policy instruments are the partitions $\Omega_{I}^{P}$ and $\Omega_{J}^{P}$ and a pair of maps $\left(H^{C O s}\left(\delta_{i}, \widehat{\delta}\right), K^{C O s}(\widehat{\delta})\right)$. We restrict the partitions to have the structure $\Omega_{I}^{e}(\widehat{\delta})=\Omega_{J}^{e}(\widehat{\delta})=[\widehat{\delta}, 1)$. Given that firms are ex-ante identical, the informational constraints embedded into the definition of $P($.$) , and the properties of the (implicit)$ matching function, to impose this structure on $\Omega_{I}^{P}$ and $\Omega_{J}^{P}$ does not entail any loss of generality. Also, observe that, given that firms are identical, expected total surplus and realized total surplus coincide.

We define an allocation Constrained Optimal (or CO) if and only if it solves the planner's optimization problem above. Let $\delta^{C O}$ be the level of the threshold associated with the $\mathrm{CO}$ allocation.

Proposition 3. Under the maintained assumptions, each economy with frictions has a CO allocation. Equilibrium allocations are never $C O$, and they are characterized by underinvestment in the amount of physical capital and in educational effort. Moreover, for each economy, there is a lower bound $\underline{c}_{I}^{e}>0$ such that, for each $c_{I}^{e}<\underline{c}_{I}^{e}, \delta^{F}<\delta^{C O}$, i.e., overinvestment in educational level holds.

Proof. See Appendix 1.

The source of inefficiency considered by Acemoglu (1996) reappears in our setup, because, given any threshold level $\widehat{\delta}, H^{C O s}\left(\delta_{i}, \widehat{\delta}\right)>\widetilde{H}^{s}\left(\delta_{i}, \widehat{\delta}\right)$, for each $\delta_{i}$, and $K^{C O s}(\widehat{\delta})>\widetilde{K}^{s}(\widehat{\delta})$. On the other hand, the relation between $\delta^{C O}$ and $\delta^{F}$ is not univocal. When the direct costs of education are sufficiently low, we always obtain $\delta^{F}<\delta^{C O}$. For sufficiently high values of $c_{I}^{e}$, however, it can be $\delta^{F}>\delta^{C O}$, as established in Example A2 in Appendix. ${ }^{18}$ Finally, one can show that, once the optimal subsidies $(\tau, \zeta)$ are introduced, to implement the $\mathrm{CO}$ allocation we always need $\Delta c_{I}^{e}>0$. Thus, $\mathrm{CO}$ always requires us to shrink the set of agents investing

\footnotetext{
${ }^{18}$ Bear in mind that, in computing $\delta^{F}$ and $\delta^{C O}$, we use different investment functions: $\left(\widetilde{H}^{s}(),. \widetilde{K}^{s}().\right)$ and $\left(H^{C O s}(),. K^{C O s}().\right)$, respectively. Hence, there is no contradiction between this property and the fact (established below) that the surplus associated with the market equilibrium is always increasing in the threshold value, even when $1>\delta^{F}>\delta^{C O}$
} 
in the high skill sector (compared to its equilibrium value in the market economy with optimal subsidies $(\tau, \zeta))$.

It is easy to see that the $\mathrm{CO}$ distribution of investments in human and physical capital can be attained with an appropriate system of subsidies to the investments, and of fixed taxes or subsidies on the direct costs of education. Moreover, given that preferences are quasi-linear, the system of tax and subsidies can be balanced using uniform lump-sum taxes $(T)$ on workers (notice that, in the absence of positive endowments of consumption goods, this could entail negative consumption for some subset of agents).

COROllary 1. There is a (balanced budget) system of taxes and subsidies $\xi$ such that the associated equilibrium allocation is $C O$.

Proof. See Appendix 1.

Remark 6. In our set-up (as well as in Acemoglu (1996)), equilibria of the economy with frictions are constrained inefficient for each value of $\beta$, because, at $\xi=0$, even if $\delta^{C O}=\delta^{F}$

$$
\frac{\widetilde{H}^{s}\left(\delta_{i}, \delta^{C O}\right)}{H^{C O s}\left(\delta_{i}, \delta^{C O}\right)}=(1-\beta)^{\frac{1-\alpha}{\alpha \Gamma}} \beta^{\frac{1}{\Gamma}} \neq 1, \text { for each } s \text { and } i,
$$

and

$$
\frac{\widetilde{K}^{s}\left(\delta^{C O}\right)}{K^{C O s}\left(\delta^{C O}\right)}=(1-\beta)^{\frac{1+\Gamma-\alpha}{\alpha \Gamma}} \beta^{\frac{1}{\Gamma}} \neq 1, \text { for each } s .
$$

In the usual random matching model, efficiency obtains when the Hosios' condition is satisfied, i.e., when $\beta$ is equal to the absolute value of the elasticity of the matching function. In our economy there is always full employment, so that no congestion externality is at work. Therefore, the Hosios' condition has no connection with Pareto efficiency. ${ }^{19}$

\subsection{The effect of income taxes and subsidies to education on total surplus}

We conclude considering the welfare effects of alternative, balanced budget, tax schemes. In particular, we study the effect on total surplus of local changes in the vector $\xi$, in a neighborhood of $\xi=0$. We just consider the effects of $\left(\tau, \Delta c_{I}^{e}\right)$. Assume that $\left.\frac{\partial \widetilde{F}}{\partial \widehat{\delta}}\right|_{\widehat{\delta}=\delta^{F}}>0$. Our first result is that an increase in the direct cost of education (redistributing the revenues as lump-sum transfer), or an increase of the subsidies to labor income in the "low skill" sector ne (financed with lump-sum taxes) always has a positive effect on total surplus. On the contrary, an increase in the subsidy to labor income in the high skill sector (again, financed with lump-sum taxes) may decrease it. The intuition behind the result is fairly simple, also given the discussion of the comparative statics of equilibria in Prop. 2 above. A subsidy $\tau^{n e}>0$ has a direct, positive incentive effect on effort in this sector, and a positive composition effect on effort in both sectors. This is because it induces an increase in the equilibrium value of $\delta^{F}(\xi)$, which, by itself, increases investments in both sectors. Due to the composition effect, a tax on higher education $\Delta c_{I}^{e}>0$ has an indirect, positive impact on effort in both sectors. Therefore, these two policies

\footnotetext{
${ }^{19}$ Given any threshold $\widehat{\delta}$, as observed in Acemoglu (1996, p. 789), the externalities are related to "the value of the future matches and are always positive".
} 
always lead to an increase in total surplus. The third one $\left(\tau^{e}>0\right)$ makes sector $e$ more attractive to workers. Therefore, it induces some workers with $\delta_{i}<\delta^{F}(0)$ to switch to sector $e$. Hence, it has an unambiguous, negative composition effect on the welfare of the workers remaining in sector ne, and on the expected profits in this sector. The negative effect on the welfare of the workers in sector $e$, due to the composition effect, may actually overcome the positive incentive effect in this sector, too. More generally, the net effect on total surplus is ambiguous, and there are economies where subsidies in the high skill sector induce a lower total surplus. This is established in Prop. 4 and by a final example. In showing these results, the main difficulty is that a change in the threshold induces a discontinuous jump in the expected producer's surplus for the firms changing sectors. We provide one sufficient condition which guarantees that, at the equilibrium, the total surplus is increasing in the value of the threshold. This condition is far from necessary for our results. The condition is that, given $(\alpha, \beta, \Gamma)$, the threshold value $\delta^{F}$ must be below some upper limit $\bar{\delta}$. Given Prop. 1, this essentially implies a lower bound on the ratio $\frac{A^{e}}{A^{n e}}$. The implicit restriction on the equilibrium threshold is not unreasonable. For instance, for $\alpha=\frac{2}{3}$, the total expected surplus is increasing in $\delta^{F}$ if $\delta^{F}<0.6$ and $\Gamma=0.2$, if $\delta^{F}<0.35$ and $\Gamma=0.5$ and so on. The critical value $\bar{\delta}$ is decreasing in $\alpha$ and $\Gamma{ }^{20}$

To conclude, let's make formal the heuristic argument above. Given $\xi$, workers and firms choose their individually optimal behavior. Let $S\left(\delta^{F}(\xi), \xi\right)$ be the expected total surplus corresponding to the equilibrium associated with the vector $\xi$ of policy instruments. Then,

$$
\begin{aligned}
S\left(\delta^{F}(\xi), \xi\right) \equiv & \sum_{s} \int_{\Omega_{J}^{s}\left(\delta^{F}(\xi)\right)} E_{\Omega_{I}^{s}\left(\delta^{F}(\xi)\right)}\left(\widetilde{\Pi}^{s}\left(\delta_{i}, \delta^{F}(\xi), \xi\right)\right) d j \\
& +\sum_{s} \int_{\Omega_{I}^{s}\left(\delta^{F}(\xi)\right)} \widetilde{V}^{s}\left(\delta_{i}, \delta^{F}(\xi), \xi\right) d i
\end{aligned}
$$

with total lump-sum taxes given by

$$
T(\xi)=\sum_{s} \tau^{s} \int_{\Omega_{I}^{s}\left(\delta^{F}(\xi)\right)} w^{s}\left(\delta_{i}, \delta^{F}(\xi), \xi\right) d i-\Delta c_{I}^{e} \nu\left(\Omega_{I}^{e}\left(\delta^{F}(\xi)\right)\right),
$$

so that the budget is balanced.

Proposition 4. Consider an equilibrium associated with $\xi=0$ and such that $\left.\frac{\partial f(.)}{\partial \widehat{\delta}}\right|_{\widehat{\delta}=\delta^{F}(0)}>0$ and

$$
E_{\Omega_{I}^{e}\left(\delta^{F}(0)\right)}\left(\delta_{i}^{1+\Gamma-\alpha}\right) \geq \frac{E_{\Omega_{I}^{n e}\left(\delta^{F}(0)\right)}\left(\delta_{i}^{\frac{\alpha}{1+\Gamma-\alpha}}\right)}{1-\alpha} .
$$

Then,

i. $\Delta c_{I}^{e}>0$, and sufficiently small, increases total surplus,

ii. $\quad \tau^{\text {ne }}>0$, and sufficiently small, increases total surplus,

iii. $\quad \tau^{e}>0$, and sufficiently small, may decrease total surplus.

\footnotetext{
${ }^{20} \mathrm{An}$ alternative sufficient condition is that $\beta$ is "large enough". Notice that, for the class of economies considered in Appendix 2, total expected surplus is increasing in $\delta^{F}$. Here, we need additional restrictions because of the discontinuity of the expected producer's surplus of the firms moving across sectors.
} 
The proofs of $(i, i i)$ are in Appendix 1, where we also establish that the welfare effect of a subsidy $\tau^{e}$ is, in general, indeterminate. The third statement is shown in Example A3, in the same Appendix, where we provide a strategy to construct economies where an increase in $\tau^{e}$ decreases total surplus.

Changes in expected surplus are our measure of welfare gains and losses. However, the different policy instruments have different implications also in terms of individual welfare. Abstract from the lump-sum taxes. An increase in the value of $\tau^{n e}$ (or of $\Delta c_{I}^{e}$ ) has a positive impact on the utility level of all the workers and on the expected surplus of each firm. On the contrary, an increase in $\tau^{e}$ has always a negative impact on the utility of all the workers in sector $n e$ (and on the expected surplus of all the firms active in this sector). It may have a positive or negative impact on utility and surplus of agents active in sector $e$.

To conclude, let's consider the policies where subsidies to effort are financed through taxes on the direct costs of education, instead of lump-sum taxes. The proof of the Corollary is a straightforward computation and, therefore, it is omitted.

Corollary 2. Consider the balanced budget policies $\left(\tau^{e}, \Delta c_{I}^{e}\right),\left(\tau^{n e}, \Delta c_{I}^{e}\right)$. Under the assumptions of Prop. 1 , and if $\Gamma$ is sufficiently small, $\left(\tau^{s}, \Delta c_{I}^{e}\right)>>(0,0)$ and sufficiently small increases total expected surplus.

The result requires the labor/effort supply to be sufficiently elastic to the wage rate. Under this restrictions, balanced budget policies with progressive income taxation $\left(\tau^{e}<0\right)$ and subsidies to the direct costs of education $\left(\Delta c_{I}^{e}<0\right)$ are welfare reducing. The impact of the composition effect of such a policy on total surplus is (under the maintained assumptions) always negative. Evidently, the impact of the incentive effect is also negative. The restriction on the value of $\Gamma$ guarantees that its impact on individual utilities is (in absolute value) larger that the positive one due to the transfer $\left|\Delta c_{I}^{e}\right|$ to agents investing in education. ${ }^{21}$

Finally, we have been considering a sector-contingent vector of subsidy rates $\left(\tau^{e}, \tau^{n e}\right)$. This is certainly an unusual feature of the policy. However, let $w^{s}\left(\delta_{i}, \delta^{F}\right)$ be agent $i$ 's labor income in sector $s$. It is easy to check that

$$
\max _{\Omega_{I}^{n e}\left(\delta^{F}\right)} w^{n e}\left(\delta_{i}, \delta^{F}\right) \leq w^{n e}\left(\delta^{F}, \delta^{F}\right)<w^{e}\left(\delta^{F}, \delta^{F}\right) \leq \min _{\Omega_{I}^{e}\left(\delta^{F}\right)} w^{e}\left(\delta_{i}, \delta^{F}\right) .
$$

Hence, given the properties of the utility functions, the same results can be obtained with a standard system of step-linear taxes or subsidies. Also, we are taking as a reference point an economy where $\xi=0$. Evidently, introducing a flat tax rate $t$ on labor incomes, we would obtain exactly the same results by changing the marginal tax rates.

\section{CONCLUSIONS}

The paper considers a class of economies where we model both extensive and intensive margins of investment choices. The main conclusion is that the results typically obtained in an efficiency unit set-up (which considers only the intensive margin) can fail to be robust to their natural extension to a Roy's model with optimal choice of investments in human and physical capital, which accounts for

\footnotetext{
${ }^{21}$ It is worthwhile to mention that these results are at variance with the very high subsidies to education and high marginal income tax rates prevailing in most European countries, see, for instance, Figure 1 in Bovemberg and Jacobs (2005).
} 
both intensive and extensive margins. By assumption, the efficiency unit framework rules out all the phenomena induced by the self-selection of the agents into different labor markets and, therefore, all the welfare consequences related to the composition effect. While an assessment of the empirical relevance of this effect is beyond the scope of this paper, from a qualitative viewpoint this is a potentially relevant phenomenon, with possible significant policy implications.

Our analysis is carried out for a simple, parametric class of economies. This allows us to compute explicitly the equilibria and the welfare effects of different policies, and to compare directly our results with the canonical results of Acemoglu (1996). Evidently, to consider quasi-linear utility function is restrictive, in particular in the analysis of the welfare impact of various policies. However, first, an extension of the analysis to a richer environment is possible, but at an high cost in terms of analytical tractability. Secondly, all the results are "open", so that they certainly survive in environments where income effects are sufficiently small. What matters most, the basic intuition behind the welfare results is strong, and they should be robust to many possible extensions of the basic set-up.

\section{APPENDIX 1}

Existence and comparative statics of the equilibria in the economy with frictions

We start with an arbitrary threshold $\widehat{\delta}$. Remember that firms are, ex-ante, identical. Then, for each firm active in $s$, the first order conditions (FOCs in the sequel) of problem $\left(\Pi^{s}\right)$ imply

$$
K^{s}\left(\widehat{\delta}, E_{\Omega_{I}^{s}(\widehat{\delta})}\left(h_{i}^{s \alpha}\right), \xi\right)=\left[\frac{(1-\beta)(1-\alpha) A^{s} E_{\Omega_{I}^{s}(\widehat{\delta})}\left(h_{i}^{s \alpha}\right)}{\mu\left(1-\zeta^{s}\right)}\right]^{\frac{1}{\alpha}}
$$

and $E_{\Omega_{J}^{s}(\widehat{\delta})}\left(K^{s}(.)^{1-\alpha}\right)=K^{s}(.)^{1-\alpha}$.

The FOCs of optimization problem $\left(U^{s}\right)$ imply that

$$
H^{s}\left(\delta_{i}, \widehat{\delta}, E_{\Omega_{J}^{s}(\widehat{\delta})}\left(k_{j}^{s 1-\alpha}\right), \xi\right)=\left[\delta_{i} \alpha \beta\left(1+\tau^{s}\right) A^{s} E_{\left.\Omega_{J}^{s} \widehat{\delta}\right)}\left(k_{j}^{s 1-\alpha}\right)\right]^{\frac{1}{1+\Gamma-\alpha}}
$$

Let $\gamma \equiv \frac{1+\Gamma}{1+\Gamma-\alpha}$, so that $(\gamma-1) \equiv \frac{\alpha}{1+\Gamma-\alpha}$.

Solving $(A 1)$ and $(A 2)$, by imposing that expectations are fulfilled, we obtain

$$
\begin{aligned}
\widetilde{K}^{s}(\widehat{\delta}, \xi)= & {\left[\frac{(1-\alpha)(1-\beta)}{\mu\left(1-\zeta^{s}\right)} E_{\left.\Omega_{I}^{s} \widehat{\delta}\right)}\left(\delta_{i}^{\gamma-1}\right)\right]^{\frac{1+\Gamma-\alpha}{\alpha \Gamma}} } \\
& \times\left(\left(1+\tau^{s}\right) \alpha \beta\right)^{\frac{1}{\Gamma}} A^{s \frac{1+\Gamma}{\alpha \Gamma}}
\end{aligned}
$$

and

$$
\begin{aligned}
\widetilde{H}^{s}\left(\delta_{i}, \widehat{\delta}, \xi\right)= & {\left[\frac{(1-\alpha)(1-\beta)}{\mu\left(1-\zeta^{s}\right)} E_{\Omega_{I}^{s}(\widehat{\delta})}\left(\delta_{i}^{\gamma-1}\right)\right]^{\frac{1-\alpha}{\alpha \Gamma}} } \\
& \times \delta_{i}^{\frac{1}{1+\Gamma-\alpha}}\left(\left(1+\tau^{s}\right) \alpha \beta\right)^{\frac{1}{\Gamma}} A^{s \frac{1}{\alpha \Gamma}}
\end{aligned}
$$


Using these functions, agent $i$ 's utility, at the $\widehat{\delta}$-conditional equilibrium and if active in sector $s$, is

$$
\begin{aligned}
\widetilde{V}^{s}\left(\delta_{i}, \widehat{\delta}, \xi\right) \equiv & U_{i}^{s}\left(\widetilde{H}^{s}\left(\delta_{i}, \widehat{\delta}, \xi\right), \widetilde{K}^{s}(\widehat{\delta}, \xi)\right)=-\left(c_{I}^{s}+\Delta c_{I}^{s}+T\right) \\
& +\left[\frac{(1-\alpha)(1-\beta)}{\mu\left(1-\zeta^{s}\right)} E_{\Omega_{I}^{s}(\widehat{\delta})}\left(\delta_{i}^{\gamma-1}\right)\right] \\
& \times \delta_{i}^{\gamma-1} \beta^{\frac{1+\Gamma}{\Gamma}} A^{s \frac{1+\Gamma}{\alpha \Gamma}}\left[\left(1+\tau^{s}\right) \alpha\right]^{\frac{1}{\Gamma}} \frac{1+\Gamma-\left(1+\tau^{s}\right) \alpha}{1+\Gamma} .
\end{aligned}
$$

Similarly, given an arbitrary $\widehat{\delta}$, firm $j$ (ex-post) surplus, if active in sector $s$ and matched with worker $i$, is

$$
\begin{aligned}
\widetilde{\Pi}^{s}\left(\delta_{i}, \widehat{\delta}, \xi\right)= & (1-\beta) A^{s \frac{1+\Gamma}{\alpha \Gamma}}\left(\left(1+\tau^{s}\right) \alpha \beta\right)^{\frac{1}{\Gamma}}\left(\delta_{i}^{\gamma-1}-(1-\alpha) E_{\Omega_{I}^{s}(\widehat{\delta})}\left(\delta_{i}^{\gamma-1}\right)\right) \\
& \times\left(\frac{(1-\alpha)(1-\beta)}{\mu\left(1-\zeta^{s}\right)} E_{\Omega_{I}^{s}(\widehat{\delta})}\left(\delta_{i}^{\gamma-1}\right)\right)^{\frac{(1+\Gamma)(1-\alpha)}{\alpha \Gamma}} .
\end{aligned}
$$

Its expected value is

$$
\begin{aligned}
E_{\Omega_{I}^{s}(\widehat{\delta})}\left(\widetilde{\Pi}^{s}\left(\delta_{i}, \widehat{\delta}, \xi\right)\right)= & {\left[\frac{(1-\alpha)(1-\beta)}{\mu\left(1-\zeta^{s}\right)} E_{\Omega_{I}^{s}(\widehat{\delta})}\left(\delta_{i}^{\gamma-1}\right)\right]^{\frac{1+\Gamma-\alpha}{\alpha \Gamma}} } \\
& \times \frac{\mu\left(1-\zeta^{s}\right) \alpha\left(\left(1+\tau^{s}\right) \alpha \beta\right)^{\frac{1}{\Gamma}} A^{s \frac{1+\Gamma}{\alpha \Gamma}}}{(1-\alpha)} .
\end{aligned}
$$

Proof of Prop. 1. Set $\xi=0$, and omit it from the notation. Pick the partition $\Omega_{I}^{P}(\widehat{\delta})$ induced by any arbitrary $\widehat{\delta}$. Assume that there is an agent $i^{\prime}$ such that $\delta_{i^{\prime}}=\widehat{\delta}$ at $\widehat{\delta}$ solving $\left(f(\widehat{\delta})-a c_{I}^{e}\right)=0$. It is easy to check that $\widetilde{F}\left(\delta_{i}, \widehat{\delta}\right) \geq 0$ if and only if $\delta_{i} \geq \widehat{\delta}$. Hence, each equilibrium partition $\Omega_{I}^{P}$ such that $\Omega_{I}^{s} \neq \emptyset$, each $s$, satisfies $\Omega_{I}^{e}\left(\delta^{F}\right)=\left[\delta^{F}, 1\right)$, as claimed in the text.

By direct computation, for each threshold $\widehat{\delta}$,

$$
E_{\Omega_{I}^{e}(\widehat{\delta})}\left(\delta_{i}^{\gamma-1}\right)=\frac{1}{\gamma} \frac{1-\widehat{\delta}^{\gamma}}{1-\widehat{\delta}} \text { and } E_{\Omega_{I}^{n e}(\widehat{\delta})}\left(\delta_{i}^{\gamma-1}\right)=\frac{\widehat{\delta}^{\gamma-1}}{\gamma} .
$$

Evidently, both functions are continuous at each $\widehat{\delta} \in(0,1)$. Given that they are conditional expectations of a strictly increasing function, both are strictly increasing in $\widehat{\delta}$. Clearly, $f(\widehat{\delta})$ is continuous at each $\widehat{\delta} \in(0,1)$. Given that $E_{\Omega_{I}^{s}(\widehat{\delta})}\left(\delta_{i}^{\gamma-1}\right)$, each $s$, is bounded, $\lim _{\widehat{\delta} \rightarrow 0} f(\widehat{\delta})=0$. Given that $\lim _{\widehat{\delta} \rightarrow 1} \frac{1-\widehat{\delta}^{\gamma}}{1-\widehat{\delta}}=\left.\frac{\partial(\widehat{\delta})}{\partial \widehat{\delta}}\right|_{\widehat{\delta}=1}=\gamma$,

$$
\lim _{\widehat{\delta} \rightarrow 1} f(\widehat{\delta})=\left(\gamma A^{e \frac{1+\Gamma}{\alpha \Gamma}}-A^{n e \frac{1+\Gamma}{\alpha \Gamma}}\right)(1+\Gamma-\alpha) \equiv \bar{C}>0 .
$$

Hence, by the intermediate value theorem, for each $c_{I}^{e}$ such that $a c_{I}^{e} \in(0, \bar{C})$, there is an interior solution to $\widetilde{F}\left(\delta^{F}, \delta^{F}\right)=0$.

Using $(A 7)$, and given that $E_{\Omega_{I}^{e}(\widehat{\delta})}\left(\delta_{i}^{\gamma-1}\right)>E_{\Omega_{I}^{n e}(\widehat{\delta})}\left(\delta_{i}^{\gamma-1}\right)$, and $A^{e}>A^{n e}$,

$$
d_{J}^{e F}=\left[E_{\Omega_{I}^{e}\left(\delta^{F}\right)}\left(\widetilde{\Pi}^{e}\left(\delta_{i}, \delta^{F}\right)\right)-E_{\Omega_{I}^{n e}\left(\delta^{F}\right)}\left(\widetilde{\Pi}^{n e}\left(\delta_{i}, \delta^{F}\right)\right)\right]>0 .
$$


Hence, all the equilibrium conditions are satisfied at $\delta^{F}$. This establishes the first part of Prop. 1.

We now proceed to study uniqueness of equilibrium and its comparative statics properties.

Observe that $\frac{\partial \widetilde{F}(.)}{\partial \Delta c_{I}^{e}}=-a<0$, and that, by direct computation,

$$
\frac{\partial f(.)}{\partial \tau^{s}}=\delta^{F \gamma-1}\left(A^{s} E_{\Omega_{I}^{s}\left(\delta^{F}\right)}\left(\delta_{i}^{\gamma-1}\right)^{(1-\alpha)}\right)^{\frac{1+\Gamma}{\alpha \Gamma}} \frac{(1+\Gamma)(1-\alpha)}{\Gamma}(-1)^{\varphi(s)}>0,
$$

with $\varphi(e)=2$ and $\varphi(n e)=1$, so that $\frac{\partial f(.)}{\partial \tau^{e}}>0$ and $\frac{\partial f(.)}{\partial \tau^{n e}}<0$. It is also easy to see that $\frac{\partial f(.)}{\partial A^{e}}>0$, while $\frac{\partial f(.)}{\partial A^{n e}}<0$. Hence, uniqueness of equilibrium, and the signs of the comparative statics properties, could be immediately established if the sign of $\left.\frac{\partial f(.)}{\partial \widehat{\delta}}\right|_{\widehat{\delta}=\delta^{F}}$ was uniquely defined. Unfortunately, this is not the case. As established in Example A1 below, there are economies with multiple equilibria and where, obviously, sign $\left|\frac{\partial f(.)}{\partial \widehat{\delta}}\right|_{\widehat{\delta}=\delta^{F}} \mid$ varies across equilibria. Hence, to establish the second part of Prop. 1, we need to impose additional restrictions on the parameter space.

By direct computation, at $\xi=0$,

$$
\begin{aligned}
& \frac{\partial f(.)}{\partial \widehat{\delta}}=(\gamma-1) \frac{1}{\widehat{\delta}} f(\widehat{\delta})+(1+\Gamma-\alpha) \frac{(1-\alpha)(1+\Gamma)}{\alpha \Gamma} \frac{\widehat{\delta}^{\gamma-1}}{\widehat{\delta}} \\
& \times\left[A^{e \frac{1+\Gamma}{\alpha \Gamma}} E_{\Omega_{I}^{e}(\widehat{\delta})}\left(\delta_{i}^{\gamma-1}\right)^{\frac{(1-\alpha)(1+\Gamma)}{\alpha \Gamma}} \eta_{\alpha}^{e}(\widehat{\delta})\right. \\
& \left.-A^{n e \frac{1+\Gamma}{\alpha \Gamma}} E_{\Omega_{I}^{n e}(\widehat{\delta})}\left(\delta_{i}^{\gamma-1}\right)^{\frac{(1-\alpha)(1+\Gamma)}{\alpha \Gamma}} \eta_{\alpha}^{n e}(\widehat{\delta})\right],
\end{aligned}
$$

where $\eta_{\alpha}^{s}(\widehat{\delta})$ is the elasticity of $E_{\Omega_{I}^{s}(\widehat{\delta})}\left(\delta_{i}^{\gamma-1}\right)$ with respect to $\widehat{\delta}$. By direct computation, $\eta_{\alpha}^{n e}(\widehat{\delta})=(\gamma-1)$, while $\eta_{\alpha}^{e}(\widehat{\delta})=\frac{-\gamma \widehat{\delta}^{\gamma}(1-\widehat{\delta})+\widehat{\delta}\left(1-\widehat{\delta}^{\gamma}\right)}{(1-\widehat{\delta})\left(1-\widehat{\delta}^{\gamma}\right)}$. With a straightforward manipulation, we obtain

$$
\begin{aligned}
& \frac{\Gamma \widehat{\delta}^{\frac{\Gamma-1}{\Gamma}}}{1+\Gamma-\alpha}\left[\frac{\gamma^{(1-\alpha)}}{A^{n e}}\right]^{\frac{1+\Gamma}{\alpha \Gamma}} \frac{\partial f(.)}{\partial \widehat{\delta}} \\
= & \left(\frac{A^{e}}{A^{n e}}\left(\frac{1-\widehat{\delta}^{\gamma}}{(1-\widehat{\delta}) \widehat{\delta}^{\gamma-1}}\right)^{(1-\alpha)}\right)^{\frac{1+\Gamma}{\alpha \Gamma}}\left(\frac{(1-\alpha)(1+\Gamma)}{\alpha} \eta_{\alpha}^{e}(\widehat{\delta})+\Gamma(\gamma-1)\right)-1 .
\end{aligned}
$$

If $\eta_{\alpha}^{e}(\widehat{\delta}) \geq 0$ at each $\widehat{\delta} \in(0,1)$, and $\frac{1-\widehat{\delta}^{\gamma}}{(1-\widehat{\delta}) \widehat{\delta}^{\gamma-1}}$ is bounded away from zero, the right hand side of the eq. above is always positive, for $\frac{A^{e}}{A^{n e}}$ sufficiently large. Therefore, for $A^{e}$ large enough, $\frac{\partial f(.)}{\partial \widehat{\delta}}>0$ at each $\widehat{\delta}$ and, in particular, at each equilibrium threshold. Evidently, if $\frac{\partial \widetilde{F}(.)}{\partial \widehat{\delta}}\left(=\frac{\partial f(.)}{\partial \widehat{\delta}}\right)>0$ at each solution to $\widetilde{F}(\widehat{\delta}, \widehat{\delta})=0$, the solution must be unique. Moreover, by the implicit function theorem, $\left.\frac{\partial f(.)}{\partial \widehat{\delta}}\right|_{\widehat{\delta}=\delta^{F}}>0$ at each equilibrium implies that $\delta^{F}($.$) satisfies \frac{\partial \delta^{F}(.)}{\partial \tau^{e}}<0, \frac{\partial \delta^{F}(.)}{\partial \tau^{n e}}>0, \frac{\partial \delta^{F(.)}}{\partial \Delta c_{I}^{e}}>0$, $\left.\frac{\partial \delta^{F(.)}}{\partial A^{e}}\right|_{\xi=0}<0$ and $\frac{\partial \delta^{F(.)}}{\partial A^{n e}}>0$, as claimed.

Hence, to conclude, we need the two additional results mentioned above (we omit the index ${ }^{1+1}$ to simplify notation): 
Fact 1. $\eta_{\alpha}^{e}(\delta) \geq 0$, at each $\delta \in(0,1)$.

By direct computation, $\eta_{\alpha}^{e}(0)=0$ and $\eta_{\alpha}^{e}(1)=\frac{\gamma-1}{2}>0$. Hence, either there is $\bar{\delta} \in(0,1)$ such that $\eta_{\alpha}^{e}(\bar{\delta})=0$ or $\eta_{\alpha}^{e}(\delta)>0$ for each $\delta \in(0,1)$, as claimed. Consider the numerator of $\eta_{\alpha}^{e}(\delta)$, call it $g(\delta)$,

$$
g(\delta)=-\gamma \delta^{\gamma}(1-\delta)+\delta\left(1-\delta^{\gamma}\right) .
$$

Given that the denominator is strictly positive for each $\delta \in(0,1), \eta^{e}(\delta) \leq 0$ if and only if $g(\delta) \leq 0$. Clearly, $g(0)=g(1)=0$. Given that

$$
\frac{\partial g(.)}{\partial \delta}=\left(1-\gamma^{2} \delta^{\gamma-1}+\left(\gamma^{2}-1\right) \delta^{\gamma}\right)
$$

$\left.\frac{\partial g(\cdot)}{\partial \delta}\right|_{\delta=0}>0$ and $\left.\frac{\partial g(\cdot)}{\partial \delta}\right|_{\delta=1}=0$. Moreover,

$$
\left.\frac{\partial^{2} g(.)}{\partial \delta^{2}}\right|_{\delta=1}=\gamma\left(\gamma^{2}-1\right) \delta^{\gamma-1}-\gamma^{2}(\gamma-1) \delta^{\gamma-2}=\gamma(\gamma-1)>0,
$$

so that $\delta=1$ is a local minimum of $g(\delta)$. Hence, if there is a $\widetilde{\delta} \in(0,1)$ such that $g(\widetilde{\delta})=0$, there must also be a $\bar{\delta} \in(0,1)$ such that $g(\bar{\delta})=0$ and $\left.\frac{\partial g(.)}{\partial \delta}\right|_{\delta=\bar{\delta}}>$ 0 . Given that, by assumption, $\bar{\delta} \in(0,1), \bar{\delta} \neq 0$, and, therefore, $\frac{g(\bar{\delta})}{\bar{\delta}}=0$, and $\left(\left.\frac{\partial g(.)}{\partial \delta}\right|_{\delta=\bar{\delta}}-\frac{g(\bar{\delta})}{\bar{\delta}}\right)>0$. However,

$$
\begin{aligned}
0 & <\left.\frac{\partial g(.)}{\partial \delta}\right|_{\delta=\bar{\delta}}-\frac{g(\bar{\delta})}{\bar{\delta}}=-\gamma^{2} \bar{\delta}^{\gamma-1}+\left(\gamma^{2}-1\right) \bar{\delta}^{\gamma}+\gamma \bar{\delta}^{\gamma-1}(1-\bar{\delta})+\bar{\delta}^{\gamma} \\
& =\left(\gamma-\gamma^{2}\right)(1-\bar{\delta}) \bar{\delta}^{\gamma-1}<0
\end{aligned}
$$

because $\gamma>1$. A contradiction. Hence, $g(\delta)>0$ and, therefore, $\eta_{\alpha}^{e}(\delta)>0$, at each $\delta \in(0,1)$.

Fact 2. Let $G(\delta) \equiv\left(\frac{1-\delta^{\gamma}}{1-\delta} \frac{\delta}{\delta^{\gamma}}\right)$. Then, $G(\delta)>\gamma>1$, for each $\delta \in(0,1)$.

The result is quite obvious from the geometrical viewpoint. Alternatively, observe that $\lim _{\delta \rightarrow 0} G(\delta)=+\infty$ and $\lim _{\delta \rightarrow 1} G(\delta)=\gamma$. Hence, to establish the Fact, it suffices to show that $\frac{\partial G(\delta)}{\partial \delta}<0$ at each $\delta \in(0,1)$. By direct computation,

$$
\left.\frac{\partial G(\delta)}{\partial \delta}\right|_{\delta=\widehat{\delta}}=\frac{\gamma}{(1-\widehat{\delta}) \widehat{\delta}^{\gamma}}\left(\frac{1}{\gamma} \frac{1-\widehat{\delta}^{\gamma}}{1-\widehat{\delta}}-1\right)=\frac{\gamma}{(1-\widehat{\delta}) \widehat{\delta}^{\gamma}}\left(E_{\Omega_{I}^{e}(\widehat{\delta})}\left(\delta_{i}^{\gamma-1}\right)-1\right)<0
$$

EXAMPLE A1. We show that there are economies such that $\left.\frac{\partial f(.)}{\partial \hat{\delta}}\right|_{\widehat{\delta}=\delta^{F}}<0$. Fix $\xi=0$. Let $\alpha=\frac{1}{2}, \Gamma=10, A^{n e}=1$, and $A^{e}=11 / 10$. By direct computation,

$$
f(\widehat{\delta})=10.5\left(\frac{105}{110}\right)^{\frac{11}{10}}\left[\widehat{\delta}^{\frac{1}{21}}\left(\frac{11}{10}\left(\frac{1-\widehat{\delta}^{\frac{110}{105}}}{1-\widehat{\delta}^{\frac{1}{2}}}\right)^{\frac{11}{5}}-\widehat{\delta}^{\frac{1}{10}}\right] .\right.
$$


$\frac{\partial f(\widehat{\delta})}{\partial \widehat{\delta}}$ is strictly positive for $\widehat{\delta}$ sufficiently small, and negative for all $\widehat{\delta}$ larger than some critical value $\bar{\delta}$. For instance, one can check that $\left.\frac{\partial f(\widehat{\delta})}{\partial \widehat{\delta}}\right|_{\widehat{\delta}=\frac{1}{2}}<0$, while $\left\{\left.\frac{\partial f(\widehat{\delta})}{\partial \hat{\delta}}\right|_{\widehat{\delta}}\right\}_{v=0}^{\infty}$ with $\widehat{\delta}^{v} \rightarrow 0$ is unbounded above. Clearly, choosing appropriately $c_{I}^{e}$, we can construct an economy with $\delta^{F}=\frac{1}{2}$, i.e., such that $\left.\frac{\partial f(\widehat{\delta})}{\partial \hat{\delta}}\right|_{\widehat{\delta}=\delta^{F}}<0$. As argued in Remark 1 , this implies that, for some values of $c_{I}^{e}$, this economy has multiple equilibria.

\section{Inefficiency properties of the economy with frictions}

The optimal choice $k_{j}^{s}$ is clearly $j$-invariant and, by assumption, $\nu\left(\Omega_{I}^{s}(\widehat{\delta})\right)=$ $\nu\left(\Omega_{J}^{s}(\widehat{\delta})\right)$. Hence, the planner's objective function can be rewritten as

$$
\begin{aligned}
P\left(h_{i}^{s}, k^{s}, \widehat{\delta}\right) \equiv & \sum_{s} \int_{\Omega_{I}^{s}(\widehat{\delta})}\left(\beta A^{s} h_{i}^{s \alpha} k^{s(1-\alpha)}-\frac{1}{\delta_{i}} \frac{h_{i}^{s(1+\Gamma)}}{1+\Gamma}\right) d i-c_{I}^{s} \nu\left(\Omega_{I}^{s}(\widehat{\delta})\right) \\
& +\sum_{s}\left((1-\beta) A^{s} \frac{\int_{\Omega_{I}^{s}(\widehat{\delta})} h_{i}^{s \alpha} d i}{\nu\left(\Omega_{I}^{s}(\widehat{\delta})\right)} k^{s(1-\alpha)}-\mu k^{s}\right) \nu\left(\Omega_{J}^{s}(\widehat{\delta})\right) \\
= & \sum_{s} \int_{\Omega_{I}^{s}(\widehat{\delta})}\left(A^{s} h_{i}^{s \alpha} k^{s(1-\alpha)}-\frac{1}{\delta_{i}} \frac{h_{i}^{s(1+\Gamma)}}{1+\Gamma}\right) d i \\
& -\left(c_{I}^{s}+\mu k^{s}\right) \nu\left(\Omega_{I}^{s}(\widehat{\delta})\right) .
\end{aligned}
$$

Its optimization problem is

$$
\max _{\left(h_{i}^{s}, k^{s}, \widehat{\delta}\right)} P\left(h_{i}^{s}, k^{s}, \widehat{\delta}\right)
$$

It is convenient to decompose $(P)$ into three problems. First, given an arbitrary value $\widehat{\delta}$, we determine the maps $\left(H^{C O s}\left(\delta_{i}, \widehat{\delta}\right), K^{C O s}(\widehat{\delta})\right)$ solving, for each $s$, the optimization problem

$$
\begin{aligned}
\max _{\left(h_{i}^{s}, k^{s}\right)} P_{\widehat{\delta}}^{s}\left(h_{i}^{s}, k^{s}\right) \equiv & \int_{\Omega_{I}^{s}(\widehat{\delta})}\left[A^{s} h_{i}^{s \alpha} k^{s(1-\alpha)}-\frac{1}{\delta_{i}} \frac{h_{i}^{s(1+\Gamma)}}{1+\Gamma}\right] d i \\
& -\left(c_{I}^{s}+\mu k^{s}\right) \nu\left(\Omega_{I}^{s}(\widehat{\delta})\right) .
\end{aligned}
$$

Next, given the value functions $P^{s}(\widehat{\delta})$ of the two problems $\left(P_{\widehat{\delta}}^{s}\right), s=n e, e$, we can recast problem $(P)$ as

$$
\max _{\widehat{\delta}} \bar{P}(\widehat{\delta}) \equiv P^{e}(\widehat{\delta})+P^{n e}(\widehat{\delta}),
$$

finding the optimal value of $\widehat{\delta}, \delta^{C O}$.

Proof of Prop. 3. Given that optimization problem $\left(P_{\widehat{\delta}}^{s}\right)$ is concave, each $s$, its solution is completely characterized by the FOCs:

i. $\quad \frac{\partial P_{\tilde{\delta}}^{s}\left(h_{i}^{s}, k^{s}\right)}{\partial h_{i}}=\alpha A^{s} k^{s(1-\alpha)} h_{i}^{s(\alpha-1)}-\frac{1}{\delta_{i}} h_{i}^{s \Gamma}=0$,

$$
\text { ii. } \quad \frac{\partial P_{\tilde{\delta}}^{s}\left(h_{i}^{s}, k^{s}\right)}{\partial k}=(1-\alpha) A^{s} k^{s(-\alpha)} \int_{\Omega_{I}^{s}(\widehat{\delta})} h_{i}^{s \alpha} d i-\mu \int_{\Omega_{I}^{s}(\widehat{\delta})} d i=0,
$$

which imply 
a. $\quad K^{C O s}(\widehat{\delta})=A^{s \frac{1+\Gamma}{\alpha \Gamma}} \alpha^{\frac{1}{\Gamma}}\left(\frac{1-\alpha}{\mu} E_{\Omega_{I}^{s}}\left(\delta_{i}^{\gamma-1}\right)\right)^{\frac{1+\Gamma-\alpha}{\alpha \Gamma}}$,

b. $\quad H^{C O s}\left(\delta_{i}, \widehat{\delta}\right)=\delta_{i}^{\frac{1}{1+\Gamma-\alpha}} \alpha^{\frac{1}{\Gamma}} A^{s \frac{1}{\alpha \Gamma}}\left(\frac{1-\alpha}{\mu} E_{\Omega_{I}^{s}}\left(\delta_{i}^{\gamma-1}\right)\right)^{\frac{1-\alpha}{\alpha \Gamma}}$.

Comparing $a-b$ to $(A 3)-(A 4), K^{C O s}(\widehat{\delta})>\widetilde{K}^{s}(\widehat{\delta})$ and $H^{C O s}\left(\delta_{i}, \widehat{\delta}\right)>\widetilde{H}^{s}\left(\delta_{i}, \widehat{\delta}\right)$, for each $\widehat{\delta}, \delta_{i}$ and $s$. Therefore, equilibria are always characterized by underinvestment in physical capital and in the effort in education. Demand and supply functions are clearly well-defined and continuous at each $\widehat{\delta} \in(0,1)$. By substituting in the objective function the optimal values $\left(K^{C O s}(\widehat{\delta}), H^{C O s}\left(\delta_{i}, \widehat{\delta}\right)\right)$, we obtain

$$
b \bar{P}(\widehat{\delta}) \equiv \frac{\alpha \Gamma}{1+\Gamma} \sum_{s} v\left(\Omega_{I}^{e}(\widehat{\delta})\right) A^{s \frac{1+\Gamma}{\alpha \Gamma}} E_{\Omega_{I}^{s}(\widehat{\delta})}\left(\delta_{i}^{\gamma-1}\right)^{\frac{1+\Gamma-\alpha}{\alpha \Gamma}}-v\left(\Omega_{I}^{e}(\widehat{\delta})\right) b c_{I}^{e}
$$

where $\frac{1}{b} \equiv \alpha^{\frac{1}{\Gamma}}\left(\frac{1-\alpha}{\mu}\right)^{\frac{(1-\alpha)(1+\Gamma)}{\alpha \Gamma}}$. Given that $\bar{P}(\widehat{\delta})$ is a continuous function, problem $(\bar{P})$ has a solution, either internal or at one of the boundary points, and, therefore, $\mathrm{CO}$ allocations exist.

Compare a market allocation and any $\mathrm{CO}$ allocation. If $\delta^{C O}=\delta^{F}=\widehat{\delta}$, $K^{C O s}(\widehat{\delta}) \neq \widetilde{K}^{s}(\widehat{\delta})$ and the market allocation is not CO. Otherwise, $\delta^{C O} \neq \delta^{F}$ and constrained inefficiency follows immediately.

To establish the second part of Prop. 3, observe that, by direct computation and rearranging terms, the (necessary) FOC of problem $(\bar{P})$ can be written as

$$
\begin{aligned}
0= & \gamma b \frac{\partial \bar{P}(\widehat{\delta})}{\partial \widehat{\delta}}=\gamma \alpha A^{n e \frac{1+\Gamma}{\alpha \Gamma}} E_{\Omega_{I}^{n e}(\widehat{\delta})}\left(\delta_{i}^{\gamma-1}\right)^{\frac{1+\Gamma-\alpha}{\alpha \Gamma}}+A^{e \frac{1+\Gamma}{\alpha \Gamma}} E_{\Omega_{I}^{e}(\widehat{\delta})}\left(\delta_{i}^{\gamma-1}\right)^{\frac{(1+\Gamma)(1-\alpha)}{\alpha \Gamma}} \\
& \times\left((1-\alpha) \frac{1-\widehat{\delta}^{\gamma}}{1-\widehat{\delta}}-\widehat{\delta}^{\gamma-1}\right)+\gamma b c_{I}^{e} .
\end{aligned}
$$

Hence, $\delta^{C O}$ is either the solution to

$$
\begin{aligned}
\gamma b c_{I}^{e}= & M^{C O}(\widehat{\delta}) \equiv A^{e \frac{1+\Gamma}{\alpha \Gamma}} E_{\Omega_{I}^{e}(\widehat{\delta})}\left(\delta_{i}^{\gamma-1}\right)^{\frac{(1+\Gamma)(1-\alpha)}{\alpha \Gamma}} \times\left(\widehat{\delta}^{\gamma-1}-(1-\alpha) \frac{1-\widehat{\delta}^{\gamma}}{1-\widehat{\delta}}\right) \\
& -\gamma \alpha A^{n e \frac{1+\Gamma}{\alpha \Gamma}} E_{\Omega_{I}^{n e}}\left(\delta_{i}^{\gamma-1}\right)^{\frac{1+\Gamma-\alpha}{\alpha \Gamma}}
\end{aligned}
$$

or $\delta^{C O} \in\{0,1\}$. The condition defining the equilibrium value $\delta^{F}$ (i.e., $\widetilde{F}(\widehat{\delta}, \widehat{\delta})=0$ ) can be recasted as

$$
\begin{aligned}
\gamma b c_{I}^{e}= & M^{F}(\widehat{\delta}) \equiv\left(\beta(1-\beta)^{\frac{1-\alpha}{\alpha}}\right)^{\frac{1+\Gamma}{\Gamma} \widehat{\delta}^{\gamma-1}} A^{e \frac{1+\Gamma}{\alpha \Gamma}} E_{\Omega_{I}^{e}(\widehat{\delta})}\left(\delta_{i}^{\gamma-1}\right)^{\frac{(1-\alpha)(1+\Gamma)}{\alpha \Gamma}} \\
& -\left(\beta(1-\beta)^{\frac{1-\alpha}{\alpha}}\right)^{\frac{1+\Gamma}{\Gamma} \widehat{\delta}^{\gamma-1}} A^{n e \frac{1+\Gamma}{\alpha \Gamma}} E_{\Omega_{I}^{n e}(\widehat{\delta})}\left(\delta_{i}^{\gamma-1}\right)^{\frac{(1-\alpha)(1+\Gamma)}{\alpha \Gamma}}
\end{aligned}
$$

Evidently, $M^{F}(0)=0$, while it is easy to verify that $M^{C O}(0)<0$. Therefore, existence of the lower bound $\underline{c}_{I}^{e}$ with the stated properties follows by continuity.

EXAMPLE A2. Let $\Gamma=\mu=A^{n e}=1$, while $\alpha=\beta=\frac{1}{2}$, and $A^{e}=2$. Using the expressions above,

$$
M^{C O}(\widehat{\delta}) \equiv 2^{4}\left(\frac{3}{4} \frac{1-\widehat{\delta}^{\frac{4}{3}}}{1-\widehat{\delta}}\right)^{2}\left(\widehat{\delta}^{\frac{1}{3}}-\frac{1}{2} \frac{1-\widehat{\delta}^{\frac{4}{3}}}{1-\widehat{\delta}}\right)-\frac{2}{3}\left(\frac{3}{4} \widehat{\delta}^{\frac{4}{3}}\right)^{3}=\frac{32}{3} c_{I}^{e}
$$


and

$$
M^{F}(\widehat{\delta}) \equiv \widehat{\delta}^{\frac{1}{3}}\left(\left(\frac{3}{4} \frac{1-\widehat{\delta}^{\frac{4}{3}}}{1-\widehat{\delta}}\right)^{2}-\frac{1}{16}\left(\frac{3}{4} \widehat{\delta}^{\frac{1}{3}}\right)^{2}\right)=\frac{32}{3} c_{I}^{e} .
$$

In Figure 1, $M^{C O}(\widehat{\delta})$ is described by the thick line, $M^{F}(\widehat{\delta})$ by the dotted one. Notice that, in the relevant range, $M^{C O}(\widehat{\delta})$ is concave, so that $M^{C O}(\widehat{\delta})-\frac{32}{3} c_{I}^{e}=0$ is also a sufficient condition for the optimal solution $\delta^{C O}$ It is clear that that $\delta^{C O}<\delta^{F}$ for $c_{I}^{e}$ sufficiently small, while $\delta^{C O}>\delta^{F}$ for $c_{I}^{e}$ large enough.

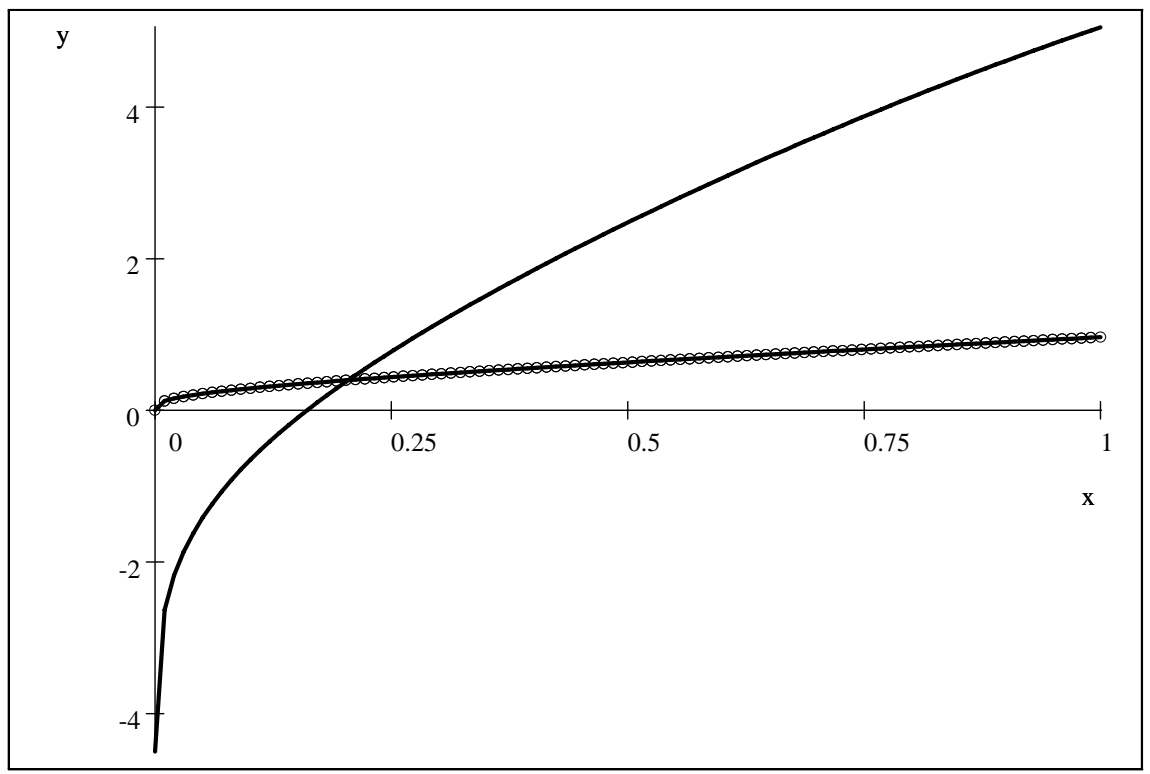

Figure 1

Proof of Corollary 1. Obviously, there are many different tax-subsidy schemes implementing the CO allocation. We will focus the analysis on linear subsidies on labor income and investments in physical capital, and on fixed fees and lump-sum taxes. Fix $\bar{\zeta}^{e}=\bar{\zeta}^{n e}=\beta$ and $\bar{\tau}^{e}=\bar{\tau}^{n e}=\frac{1-\beta}{\beta}$. It is easy to check that, given any threshold value $\widehat{\delta}$, the FOCs of the individual optimization problem in the actual economy imply that the FOCs of the (constrained) planner's optimization problem are satisfied. Let $\delta^{F}(\xi)$ be the market threshold value associated with $\xi$.

By direct computation, at the CO allocation, expected profits are zero in both sectors. Hence, firms are indifferent among sectors. Therefore, at each optimal solution $\widehat{\delta} \in(0,1)$, the FOCs of optimization problem $(\bar{P})$ are simply given by

$$
\begin{aligned}
& -\left[U_{i}^{e}\left(H^{C O e}\left(\delta_{i}=\widehat{\delta}, \widehat{\delta}\right), K^{C O e}(\widehat{\delta})\right)-U_{i}^{n e}\left(H^{C O n e}\left(\delta_{i}=\widehat{\delta}, \widehat{\delta}\right), K^{C O n e}(\widehat{\delta})\right)-c_{I}^{e}\right] \\
& +\sum_{s} \int_{\Omega_{I}^{s}(\widehat{\delta})} \frac{\partial U_{i}^{s}\left(H^{C O s}\left(\delta_{i}=\widehat{\delta}, \widehat{\delta}\right), K^{C O s}(\widehat{\delta})\right)}{\partial \widehat{\delta}}=0 .
\end{aligned}
$$

Set

$$
\overline{\Delta c}_{I}^{e}=\sum_{s} \int_{\Omega_{I}^{s}(\widehat{\delta})} \frac{\partial U_{i}^{s}\left(H^{C O s}\left(\delta_{i}=\widehat{\delta}, \widehat{\delta}\right), K^{C O s}(\widehat{\delta})\right)}{\partial \widehat{\delta}} .
$$


Then, given education fees equal to $\left(c_{I}^{e}+\overline{\Delta c}_{I}^{e}\right), \delta^{F}(\xi)=\delta^{C O}$ and the equilibrium level of total surplus coincides with its $\mathrm{CO}$ level. Finally, redistribute the total net revenues (or costs) of the fee-subsidy scheme across workers using $i$-invariant lumpsum taxes, so to balance the budget.

Proof of Prop. 4. Using the properties of the two sets $\Omega_{I}^{s}\left(\delta^{F}(\xi)\right)$ and $\Omega_{J}^{s}\left(\delta^{F}(\xi)\right)$, we can rewrite $S\left(\delta^{F}(\xi), \xi\right)$ as

$S\left(\delta^{F}(\xi), \xi\right) \equiv \sum_{s}\left(\int_{\Omega_{I}^{s}\left(\delta^{F}(\xi)\right)} \widetilde{V}^{s}\left(\delta_{i}, \delta^{F}(\xi), \xi\right) d i+\int_{\Omega_{J}^{s}\left(\delta^{F}(\xi)\right)} E_{\Omega_{I}^{s}\left(\delta^{F}(\xi)\right)}\left(\widetilde{\Pi}^{s}\left(\delta_{i}, \delta^{F}(\xi), \xi\right) d j\right)\right)$.

Remember that the net sum of taxes and subsidies is zero. Therefore,

$$
\begin{aligned}
\frac{\partial S(.)}{\partial \tau^{s}}= & \frac{\partial S(.)}{\partial \delta^{F}} \frac{\partial \delta^{F}(\xi)}{\partial \tau^{s}}+\sum_{s} \int_{\Omega_{I}^{s}\left(\delta^{F}(\xi)\right)} \frac{\partial \widetilde{V}^{s}\left(\delta_{i}, \delta^{F}(\xi), \xi\right)}{\partial \tau^{s}} d i \\
& +\sum_{s} \int_{\Omega_{J}^{s}\left(\delta^{F}(\xi)\right)} \frac{\partial E_{\Omega_{I}^{s}\left(\delta^{F}(\xi)\right)}\left(\widetilde{\Pi}^{s}\left(\delta_{i}, \delta^{F}(\xi), \xi\right)\right)}{\partial \tau^{s}} d j \\
\frac{\partial S(.)}{\partial \Delta c_{I}^{e}}= & \frac{\partial S(.)}{\partial \delta^{F}} \frac{\partial \delta^{F}(\xi)}{\partial \Delta c_{I}^{e}} .
\end{aligned}
$$

From eqs. $(A 5)$ and $(A 7)$, the last two terms of $\frac{\partial S(.)}{\partial \tau^{s}}$ are positive. By direct computation,

$$
\begin{aligned}
\frac{\partial S(.)}{\partial \delta^{F}}= & -\left[\widetilde{V}^{e}\left(\delta_{i}=\delta^{F}(\xi), \delta^{F}(\xi), \xi\right)-\widetilde{V}^{n e}\left(\delta_{i}=\delta^{F}(\xi), \delta^{F}(\xi), \xi\right)\right] \\
& -\left[\widetilde{\Pi}^{e}\left(\delta_{i}=\delta^{F}(\xi), \delta^{F}(\xi), \xi\right)-\widetilde{\Pi}^{n e}\left(\delta_{i}=\delta^{F}(\xi), \delta^{F}(\xi), \xi\right)\right] \\
& +\sum_{s} \int_{\Omega_{I}^{s}\left(\delta^{F}(\xi)\right)} \frac{\partial \widetilde{V}^{s}(.)}{\partial \delta^{F}} d i+\sum_{s} \int_{\Omega_{J}^{s}\left(\delta^{F}(\xi)\right)} \frac{\partial E_{\Omega_{I}^{s}\left(\delta^{F}\right)}\left(\widetilde{\Pi}^{s}(.)\right)}{\partial \delta^{F}} d j .
\end{aligned}
$$

By definition of $\delta^{F}(\xi)$, the first term in square brackets is zero. We have already established (see eqs. (7)) that the last four terms are positive. $\widetilde{\Pi}^{n e}\left(\delta_{i}=\right.$ $\left.\delta^{F}(\xi), \delta^{F}(\xi), \xi\right)$ is also positive, because

$$
\widetilde{\Pi}^{n e}\left(\delta_{i}=\delta^{F}(\xi), \delta^{F}(\xi), \xi\right) \geq E_{\Omega_{I}^{n e}\left(\delta^{F}\right)}\left(\widetilde{\Pi}^{n e}(.)\right) \geq 0 .
$$

Hence, a sufficient condition for $\frac{\partial S(.)}{\partial \delta^{F}}>0$ is

$$
\begin{aligned}
0 \leq & \Delta S^{e} \equiv \int_{\Omega_{I}^{e}\left(\delta^{F}(\xi)\right)} \frac{\partial \widetilde{V}^{e}(.)}{\partial \delta^{F}} d i+\int_{\Omega_{J}^{e}\left(\delta^{F}(\xi)\right)} \frac{\partial E_{\Omega_{I}^{e}\left(\delta^{F}\right)}\left(\widetilde{\Pi}^{s}(.)\right)}{\partial \delta^{F}} d j \\
& -\widetilde{\Pi}^{e}\left(\delta_{i}=\delta^{F}(\xi), \delta^{F}(\xi), \xi\right) .
\end{aligned}
$$

Define

$$
B^{e}=\left[\frac{1}{\gamma} A^{e \frac{1+\Gamma}{\alpha \Gamma}}(\alpha \beta)^{\frac{1}{\Gamma}}\left(\frac{(1-\alpha)(1-\beta)}{\mu} E_{\Omega_{I}^{e}\left(\delta^{F}\right)}\left(\delta_{i}^{\gamma-1}\right)\right)^{\frac{(1+\Gamma)(1-\alpha)}{\alpha \Gamma}}\right] .
$$


Using $\gamma \equiv \frac{1+\Gamma}{1+\Gamma-\alpha}$, and $(A 6)$, the ex-post profits of the firm matched with the worker with $\delta_{i}=\delta^{F}$ are

$$
\widetilde{\Pi}^{e}\left(\delta_{i}=\delta^{F}(\xi), \delta^{F}(\xi), \xi\right)=(1-\beta)\left(\gamma \delta^{F}(\xi)^{\gamma-1}-(1-\alpha)\left(\frac{1-\delta^{F}(\xi)^{\gamma}}{1-\delta^{F}(\xi)}\right)\right) \times B^{e} .
$$

Using $(A 5)$ and $(A 7)$,

$$
\int_{\Omega_{I}^{e}\left(\delta^{F}(\xi)\right)} \frac{\partial \widetilde{V}_{i}^{e}(.)}{\partial \delta^{F}} d i=B^{e} \frac{(1+\Gamma)(1-\alpha)}{\gamma \alpha \Gamma} \beta\left(\frac{1-\delta^{F}(\xi)^{\gamma}}{1-\delta^{F}(\xi)}-\gamma \delta^{F}(\xi)^{\gamma-1}\right)
$$

and

$$
\int_{\Omega_{J}^{e}\left(\delta^{F}(\xi)\right)} \frac{\partial E_{\Omega_{j}^{e}\left(\delta^{F}\right)}\left(\widetilde{\Pi}^{e}(.)\right)}{\partial \delta^{F}} d j=\frac{1-\alpha}{\Gamma} B^{e}(1-\beta)\left(\frac{1-\delta^{F}(\xi)^{\gamma}}{1-\delta^{F}(\xi)}-\gamma \delta^{F}(\xi)^{\gamma-1}\right) .
$$

Hence,

$$
\begin{aligned}
\frac{\Gamma \Delta S^{e}}{(1+\Gamma) \delta^{F}(\xi)^{\gamma-1} B^{e}}= & {\left[\beta \frac{(1-\alpha)}{\gamma \alpha}\left(\frac{1-\delta^{F}(\xi)^{\gamma}}{\left(1-\delta^{F}(\xi)\right) \delta^{F}(\xi)^{\gamma-1}}-\gamma\right)\right] } \\
& +(1-\beta)\left[(1-\alpha) \frac{1-\delta^{F}(\xi)^{\gamma}}{\left(1-\delta^{F}(\xi)\right) \delta^{F}(\xi)^{\gamma-1}}-1\right]>0
\end{aligned}
$$

The first term in square brackets is strictly positive (because, as shown in Fact 2 above, $\frac{1-\delta^{F}(\xi)^{\gamma}}{\left(1-\delta^{F}(\xi)\right) \delta^{F}(\xi)^{\gamma-1}}$ is bounded below by $\left.\gamma\right)$. By assumption, the second term is positive. Notice that the inequality is always satisfied for $\beta$ large enough.

When $\left.\frac{\partial f(.)}{\partial \widehat{\delta}}\right|_{\widehat{\delta}=\delta^{F}}>0, \frac{\partial \delta^{F}(.)}{\partial \Delta c_{I}^{e}}>0$ and $\frac{\partial \delta^{F}(.)}{\partial \tau^{n e}}>0$, so that $\frac{\partial S(.)}{\partial \tau^{n e}}>0$ and $\frac{\partial S(.)}{\partial \Delta c_{I}^{e}}>0$. It follows that a subsidy to labor income in sector ne, and/or an increase in the fixed cost of education $c_{I}^{e}$, increases the expected total surplus .

On the other hand, $\frac{\partial \delta^{F}(.)}{\partial \tau^{e}}<0$ and, therefore, under the maintained assumptions, the sign of $\frac{\partial S(.)}{\partial \tau^{e}}$ is undefined.

EXAMPLE A3: Welfare-reducing subsidies to investments in human capital in the high skill sector.

From eq. (2), the sign of $\left.\frac{\partial f}{\partial \hat{\delta}}\right|_{\widehat{\delta}=\delta^{F}(.)}$ depends upon the parameters $\left(\frac{A^{e}}{A^{n^{e}}}, \alpha, \Gamma\right)$, and the equilibrium level $\delta^{F}($.$) , while it doesn't depend directly on \beta$. Moreover, given $\left(\frac{A^{e}}{A^{e}}, \alpha, \Gamma\right)$, the effect of changes of $\beta$ on the value of $\delta^{F}($.$) can always be$ neutralized by appropriate changes in the parameter $c_{I}^{e}$.

Clearly,

$$
\begin{aligned}
\frac{\partial S(.)}{\partial \tau^{e}}= & \frac{\partial S(.)}{\partial \delta^{F}} \frac{\partial \delta^{F}(\xi)}{\partial \tau^{e}}+\sum_{s} \int_{\Omega_{I}^{s}\left(\delta^{F}(\xi)\right)} \frac{\partial \widetilde{V}^{s}\left(\delta_{i}, \delta^{F}(\xi), \xi\right)}{\partial \tau^{s}} d i \\
& +\sum_{s} \int_{\Omega_{J}^{s}\left(\delta^{F}(\xi)\right)} \frac{\partial E_{\Omega_{I}^{s}\left(\delta^{F}(\xi)\right)}\left(\widetilde{\Pi}^{s}\left(\delta_{i}, \delta^{F}(\xi), \xi\right)\right)}{\partial \tau^{s}} d j
\end{aligned}
$$

We want to construct an economy such that, at the equilibrium, $\frac{\partial S(.)}{\partial \tau^{e}}<0$. The last four terms of $\frac{\partial S(.)}{\partial \tau^{e}}$ are positive, and they are easily seen to be bounded above 
(looking at their parametric structure in $(A 5)$ and $(A 7)$ ). Hence, the required result is established if we can construct an equilibrium with $\frac{\partial S(.)}{\partial \delta^{F}} \frac{\partial \delta^{F}(\xi)}{\partial \tau^{e}}<0$ and arbitrarily large in absolute value. As established in Example A1 in Appendix 1, for $\frac{A^{e}}{A^{n^{e}}}$ sufficiently small, there are economies such that $\frac{\partial f(.)}{\partial \widehat{\delta}}>0$, for $\widehat{\delta}$ sufficiently small and $\frac{\partial f(.)}{\partial \hat{\delta}}<0$ for $\widehat{\delta}$ large enough. Given that the function $\frac{\partial f(.)}{\partial \hat{\delta}}$ is continuous, this implies that, for economies in this set, there is $\bar{\delta}$ such that $\left.\frac{\partial f(.)}{\partial \hat{\delta}}\right|_{\widehat{\delta}=\bar{\delta}}=0$ and $\frac{\partial f(.)}{\partial \hat{\delta}}>$ 0 at each $\widehat{\delta}<\bar{\delta}$. Given the values of all the parameters, but the actual direct cost of education, $c_{I}^{e}$, pick a sequence $\left\{c_{I}^{e v}\right\}_{v=1}^{\infty}$ such that $c_{I}^{e v}<\bar{c}_{I}^{e}=\frac{f(\bar{\delta}, \xi)}{a}$, for each $v$, and $c_{I}^{e v} \rightarrow \bar{c}_{I}^{e}$. Let $\left\{\delta^{F}\left(c_{I}^{e v}, \xi\right)\right\}_{v=1}^{\infty}$ be the associated sequence of equilibrium thresholds. By construction, there is a subsequence with $\delta^{F}\left(c_{I}^{e v}, \xi\right)<\bar{\delta}$, and $\delta^{F}\left(c_{I}^{e v}, \xi\right) \rightarrow \bar{\delta}$. Therefore, $\left.\frac{\partial f(.)}{\partial \hat{\delta}}\right|_{\widehat{\delta}=\delta^{F}\left(c_{I}^{e v}, \xi\right)}>0$, for each $v$, and $\left.\lim _{v \rightarrow \infty} \frac{\partial f(.)}{\partial \hat{\delta}}\right|_{\widehat{\delta}=\delta^{F}\left(c_{I}^{e v}, \xi\right)}=0$. By the implicit function theorem, $\frac{\partial \delta^{F}(.)}{\partial \tau^{e}}=-\frac{\frac{\partial f(.)}{\partial \tau^{e}}}{\left.\frac{\partial f(.)}{\partial \hat{\delta}}\right|_{\hat{\delta}=\delta^{F}(\xi)}}$. Given that $\frac{\partial f(.)}{\partial \tau^{e}}$ is positive and bounded away from zero (see Prop. 1), the sequence $\frac{\partial \delta^{F}(.)}{\partial \tau^{e}}$ associated with $\left\{\delta^{F}\left(c_{I}^{e v}, \xi\right)\right\}_{v=1}^{\infty}$ is negative and divergent.

To prove our result we still need to show that there are economies such that, in a neighborhood of $\bar{\delta},\left.\frac{\partial S(.)}{\partial \hat{\delta}}\right|_{\widehat{\delta}=\delta^{F}(\xi)} \geq \varepsilon$, for some $\varepsilon>0$. In the proof of Prop. 4, we have defined the expression $\Delta S^{e}$, governing the sign of $\left.\frac{\partial S(.)}{\partial \widehat{\delta}}\right|_{\widehat{\delta}=\delta^{F}(\xi)}$. It is easy to check that, given $\delta^{F}(\xi), \Delta S^{e}$ is strictly positive for $\beta$ close enough to 1 . Pick a value of $\beta, \bar{\beta}$, such that, at $\bar{\delta}, \frac{\partial S(.)}{\partial \hat{\delta}}>\varepsilon$, for some $\varepsilon>0$. By continuity, $\frac{\partial S(.)}{\partial \hat{\delta}}>\varepsilon$ at each $\delta$ close enough to $\bar{\delta}$. Change the sequence $\left\{c_{I}^{e v}\right\}_{v=1}^{\infty}$ to neutralize the change in $\delta^{F}\left(c_{I}^{e v}, \xi\right)$ due to the new value of $\beta$. For the economy so constructed, for some $c_{I}^{e}$, it must be $\frac{\partial S(.)}{\partial \tau^{e}}<0$ and arbitrarily large in absolute value, as claimed.

\section{APPENDIX 2: COMPETITIVE SPOT LABOR MARKET}

In this final Appendix, we briefly sketch a model with asymmetric information on the characteristics of the workers facing a given set of firms, and lack of contractibility of investments. These two features, together with self-selection into the high and low skill sectors, determine the welfare properties of equilibria, characterized by overinvestment in educational level. Contrary to what we have seen in the text, contingent on the equilibrium threshold, investments are at their (constrained) efficient level, because no hold-up problem is at play in this reformulation of the model.

There is a continuum of separated islands, denoted by $\ell \in(0,1)$. On each island there is an interval $(0,1)$ of identical workers and firms. Firms (denoted by a pair $(j, \ell) \in(0,1) \times(0,1))$ are identical across islands. Workers (denoted by a pair $(i, \ell) \in(0,1) \times(0,1))$ are identical within an island (i.e., with respect to the index $\ell$ ), but heterogeneous across islands, because of the parameter $\delta_{i}$, whose realization in a given island is private information of the workers. ${ }^{22}$ First, firms and workers choose the type and amount of their investments. Next, investments are mutually observable, (island specific) labor markets open and clear at the competitive

\footnotetext{
${ }^{22}$ In the sequel we implicitly assume that the realization of $E_{\Omega_{\ell}^{s}(\widehat{\delta})}\left(\delta_{i}^{\gamma-1}\right)$ coincides with its theoretical value. Using appropriate assumptions on the random variables $\delta_{i}$, this can be guaranteed.
} 
wage. Given that, ex-ante, the realization $\delta_{i}$ is not observable, firms choose their investments taking into account the (conditional on $\widehat{\delta}$ ) distribution of the human capital of the workers. Preferences and production functions are as above. Given that firms are identical, they all have the same optimal level of investments in each sector.

Each worker chooses her behavior solving: given the equilibrium maps $\left(w_{i}^{s}\left(\delta_{i}\right), s_{\ell}(j)\right)$ and the equilibrium threshold value $\bar{\delta}$,

choose $\left\{s\left(\delta_{i}, \bar{\delta}\right), H^{s}\left(\delta_{i}, \bar{\delta}\right)\right\} \in \arg \max _{s}\left\{\max _{h_{i \ell}^{s}} E_{\Omega_{J \ell}^{s}(\bar{\delta})}\left(U_{i \ell}^{s}\left(C_{i \ell}^{s}, h_{i \ell}^{s}\right)\right)\right.$ s.to $\left.C_{i \ell}^{s}=w_{i}^{s}\left(\delta_{i}\right) h_{i \ell}^{s}-c_{I}^{s}\right\}$.

where $s\left(\delta_{i}\right) \in\{n e, e\}$ denotes her choice of the optimal sector.

Given the equilibrium maps $\left(w_{i}\left(\delta_{i}\right), s\left(\delta_{i}, \bar{\delta}\right)\right)$ and the equilibrium threshold value $(\bar{\delta})$, each firm solves optimization problem

choose $\left\{s(\bar{\delta}),\left(K^{s}(\bar{\delta}), H^{s}(\bar{\delta})\right)\right\} \in \arg \max _{s}\left\{\max _{\left(k_{j \ell}^{s}, h_{j \ell}^{s}\right)} E_{\Omega_{I \ell}^{s}(\bar{\delta})}\left(A^{s} h_{j \ell}^{s \alpha} k_{j \ell}^{s(1-\alpha)}-w_{i}^{s}\left(\delta_{i}\right) h_{j \ell}^{s}-\mu k_{j \ell}^{s}\right)\right\}$,

with $s(\bar{\delta}) \in\{n e, e\}$, and where we omit the indexes $j \ell$ because the firms' choices just depend upon $s$. Bear in mind that (at the equilibrium) expected profits are always zero, so that firms are indifferent among the sector they are active in

Definition 2. A rational expectations equilibrium is a pair of maps $\left(\bar{w}_{i}^{n e}\left(\delta_{i}, \bar{\delta}\right), \bar{w}_{i}^{e}\left(\delta_{i}, \bar{\delta}\right)\right)$, a threshold value $\bar{\delta}$, and maps $\left\{s\left(\delta_{i}, \bar{\delta}\right), H^{s}\left(\delta_{i}, \bar{\delta}\right)\right\}$ and $\left\{s(\bar{\delta}), K^{s}(\bar{\delta}), K^{s}(\bar{\delta})\right\}$ such that

i. $\quad E_{\Omega_{J \ell}^{e}(\bar{\delta})}\left(U_{i \ell}^{e}\left(\bar{C}_{i}^{e}, H^{e}\left(\delta_{i}, \bar{\delta}\right)\right)\right)-E_{\Omega_{J \ell}^{n e}(\bar{\delta})}\left(U_{i \ell}^{n e}\left(\bar{C}_{i}^{n e}, H^{n e}\left(\delta_{i}, \bar{\delta}\right)\right)\right) \geq 0$ if and only if $\delta_{i} \geq \bar{\delta}$,

ii. $\quad$ for each $(i, \ell),\left\{s\left(\delta_{i}, \bar{\delta}\right), H^{s}\left(\delta_{i}, \bar{\delta}\right)\right\}$ solves $(U 2)$,

iii. $\quad$ for each $\left.j \ell,\left\{s(\bar{\delta}), K^{s}(\bar{\delta}), H^{s}(\bar{\delta})\right)\right\}$ solves $(\Pi 2)$,

iv. $\quad$ for each $\ell, \int_{(0,1)} H^{s}\left(\delta_{i}, \bar{\delta}\right) d i=\int_{(0,1)} H^{s}(\bar{\delta}) d j$.

We start solving for the ex-post competitive equilibrium, contingent on the aggregate investments in physical capital, $\bar{K}^{s}$. A straightforward computation shows that the wage map is given by

$$
w^{s}\left(\delta_{i}, \bar{K}^{s}\right)=\frac{\left(\alpha A^{s} \bar{K}^{s}(1-\alpha)\right)^{\frac{\Gamma}{1+\Gamma-\alpha}}}{\delta_{i}^{\frac{1-\alpha}{1+\Gamma}}} .
$$

Given an arbitrary threshold value $\widehat{\delta}$, ex-ante, expected profits of a firm active in sector $s$ are given by

$$
E_{\Omega^{s}(\widehat{\delta})}\left(\Pi^{s}(.)\right)=\frac{1-\alpha}{\alpha}\left(\alpha A^{s}\right)^{\frac{1}{1-\alpha}} E_{I_{\ell}(\bar{\delta})}\left(\frac{1}{w^{s}\left(\delta_{i}, \bar{K}^{s}\right)^{\frac{\alpha}{1-\alpha}}}\right) k_{j \ell}-\mu k_{j \ell},
$$

so that the zero expected profit condition imposes

$$
\frac{1-\alpha}{\mu \alpha}\left(\alpha A^{s}\right)^{\frac{1}{1-\alpha}} E_{\Omega_{I \ell}^{s}(\bar{\delta})}\left(\frac{1}{w^{s}\left(\delta_{i}, \bar{K}^{s}\right)^{\frac{\alpha}{1-\alpha}}}\right)=1 .
$$


Replacing $w^{s}\left(\delta_{i}, \bar{K}^{s}\right)$ into this condition, we obtain

$$
\widetilde{K}^{s}(\widehat{\delta})=\left(\frac{1-\alpha}{\mu}\right)^{\frac{1+\Gamma-\alpha}{\alpha \Gamma}} \alpha^{\frac{1}{\Gamma}} A^{s \frac{1+\Gamma}{\alpha \Gamma}} E_{\Omega_{I \ell}^{s}(\bar{\delta})}\left(\delta_{i}^{\gamma-1}\right)^{\frac{1+\Gamma-\alpha}{\alpha \Gamma}} .
$$

Replacing this condition into $w^{s}\left(\delta_{i}, \bar{K}^{s}\right)$, we obtain the $\widehat{\delta}$-conditional equilibrium map

$$
\bar{w}^{s}\left(\delta_{i}, \widehat{\delta}\right)=\frac{\left(\alpha A^{s}\right)^{\frac{1}{\alpha}}\left(\frac{1-\alpha}{\mu \alpha}\right)^{\frac{1-\alpha}{\alpha}} E_{\Omega_{I \ell}^{s}(\bar{\delta})}\left(\delta_{i}^{\gamma-1}\right)^{\frac{1-\alpha}{\alpha}}}{\delta_{i}^{\frac{1-\alpha}{1+\Gamma-\alpha}}} .
$$

Finally, given $\widehat{\delta}, i$ 's supply of effort (if active in sector $s$ and conditional on $\widehat{\delta}$ ) is

$$
\widetilde{H}^{s}\left(\delta_{i}, \widehat{\delta}\right)=\delta_{i}^{\frac{1}{1+\Gamma-\alpha}} \alpha^{\frac{1}{\Gamma}} A^{s \frac{1}{\alpha \Gamma}}\left[\frac{1-\alpha}{\mu} E_{\Omega_{I \ell}^{s}(\bar{\delta})}\left(\delta_{i}^{\gamma-1}\right)\right]^{\frac{1-\alpha}{\alpha \Gamma}},
$$

so that the value of the indirect utility function (if $s$ ) is

$$
\widetilde{V}^{s}\left(\delta_{i}, \widehat{\delta}\right)=\frac{\Gamma}{1+\Gamma}\left(\left(\frac{1-\alpha}{\mu \alpha} E_{\Omega_{I \ell}^{s}(\widehat{\delta})}\left(\delta_{i}^{\gamma-1}\right)\right)^{(1-\alpha)} \alpha A^{s}\right)^{\frac{(1+\Gamma)}{\alpha \Gamma}} \delta_{i}^{\frac{\alpha}{1+\Gamma-\alpha}}-c_{I}^{s} \cdot \backslash
$$

The map defining the equilibrium threshold is then

$$
\begin{aligned}
0= & \widehat{\delta}^{\frac{\alpha}{1+\Gamma-\alpha}}\left(A^{e} E_{\Omega_{I \ell}^{s}(\widehat{\delta})}\left(\delta_{i}^{\gamma-1}\right)^{(1-\alpha)}\right)^{\frac{(1+\Gamma)}{\alpha \Gamma}} \\
& -\widehat{\delta}^{\frac{\alpha}{1+\Gamma-\alpha}}\left(A^{n e} E_{\Omega_{I \ell}^{s}(\widehat{\delta})}\left(\delta_{i}^{\gamma-1}\right)^{(1-\alpha)}\right)^{\frac{(1+\Gamma)}{\alpha \Gamma}}-b c_{I}^{e},
\end{aligned}
$$

with $b=\frac{1+\Gamma}{\Gamma\left(\frac{1-\alpha}{\mu \alpha}\right)^{\frac{(1-\alpha)(1+\Gamma)}{\alpha \Gamma}} \alpha^{\frac{1+\Gamma}{\alpha \Gamma}}}$.

Modulo a multiplicative term, this expression is identical to eq. (2) in the text. Evidently, the qualitative properties of equilibria are identical in the two classes of economies.

There is, however, an important difference with respect to efficiency. According to the definition of $\mathrm{CO}$ introduced above, in this set-up, the supply of human and physical capital is $\mathrm{CO}$, given that $\widetilde{K}^{s}(\widehat{\delta})$ and $\widetilde{H}^{s}\left(\delta_{i}, \widehat{\delta}\right)$ coincide with the maps $\left\{H^{C O s}\left(\delta_{i}, \widehat{\delta}\right), K^{C O}(\widehat{\delta})\right\}$ computed in the proof of Prop. 3. Hence, perfect competition in the spot labor markets eliminates the distortion related to the hold-up problem. It follows that, if $\delta^{F}=\delta^{C O}$, the market equilibrium is CO. However, it is easy to see that it is always $\delta^{F}<\delta^{C O}$, i.e., there is always overinvestment in the education level. Indeed, given that expected profits are always zero, the planner's objective function reduces to

$$
P(\widehat{\delta})=\int_{0}^{\widehat{\delta}} \widetilde{V}^{n e}\left(\delta_{i}, \widehat{\delta}\right) d i+\int_{\widehat{\delta}}^{1} \widetilde{V}^{e}\left(\delta_{i}, \widehat{\delta}\right) d i,
$$

with FOC

$$
\begin{aligned}
\frac{\partial P(\widehat{\delta})}{\partial \widehat{\delta}}= & -\left[\widetilde{V}^{e}\left(\delta_{i}=\widehat{\delta}, \widehat{\delta}\right)-\widetilde{V}^{n e}\left(\delta_{i}=\widehat{\delta}, \widehat{\delta}\right)\right] \\
& +\int_{0}^{\widehat{\delta}} \frac{\partial \widetilde{V}^{n e}\left(\delta_{i}, \widehat{\delta}\right)}{\partial \widehat{\delta}} d i+\int_{\widehat{\delta}}^{1} \frac{\partial \widetilde{V}^{e}\left(\delta_{i}, \widehat{\delta}\right)}{\partial \widehat{\delta}} d i
\end{aligned}
$$


The last two terms are always strictly positive. Given that, by definition of $\delta^{F}$, the term in square brackets is zero, at $\delta^{F}, \frac{\partial P(\widehat{\delta})}{\partial \hat{\delta}}>0$. Hence, $\delta^{C O}>\delta^{F}$, so that we have always overinvestment in education level, at the equilibrium.

\section{REFERENCES}

Acemoglu, D.T., 1996, A microfoundation for social increasing returns in human capital accumulation, Quarterly Journal of Economics, 111, 779-804.

Acemoglu, D., and J. Angrist, 2001, How large are the social returns to education? Evidence from compulsory schooling laws, in B. Bernanke and K. Rogoff (eds.), NBER Macroeconomic Annual 2000, Cambridge MA, MIT Press, 9-58.

Acemoglu, D., and R. Shimer, 1999, Holdups and efficiency with search frictions, International Economic Review, 40, 827849.

Anderberg, D., 2008, Optimal Policy and the Risk-Properties of Human Capital Reconsidered, CESifo WPaper 2451.

Anderberg, D., and F. Andersson, 2003, Investments in human capital, wage uncertainty, and public policy, Journal of Public Economics, 87, 1521-1537.

Ben-Porath, Y., 1970, The production of human capital over time, in W. Hansen (ed.), Education, Income and Human Capital, NBER, 129-154.

Blankenau, W., 2005, Public schooling, college subsidies and growth, Journal of Economic Dynamics and Control, 29, 487-507.

Blankenau, W., and G. Camera, 2006, A simple economic theory of skill accumulation and schooling decisions, Review of Economic Dynamics 9, 93-115.

Blankenau, W., and G. Camera, 2009, Public spending on education and the incentives to student achievement, Economica, forthcoming.

Boskin, M.J., 1975, Notes on the tax treatment of human capital, NBER WP 116.

Bovenberg, A.L., and B. Jacobs, 2005, Redistribution and education subsidies are Siamese twins, Journal of Public Economics, 89, 2005-2035.

Carneiro, P., J.J. Heckman, and E. Vytlacil, 2001, Estimating the return to education when it varies among individuals, mimeo.

Caucutt, E.M., and K.B. Kumar, 2003, Higher education subsidies and heterogeneity. A dynamic analysis, Journal of Economic Dynamics and Control, 27, 1459-1502.

Charlot, O. and B. Decreuse, 2005, Self-selection in education with matching frictions, Labour Economics 12, 251-267.

Da Costa, C.E., and Maestri, L.J., 2007, The Risk Properties of Human Capital and the Design of Government Policies, European Economic Review, 51, 695-713.

De la Fuente, A., 2003, Human Capital in a Global and Knowledge-Based Economy. Part II: Assessment at the EU Country Level. Final Report, European Commission, Directorate General for Employment and Social Affairs.

Decreuse, B., 2008, Choosy search and the mismatch of talents, International Economic Review, 49, 1067-1089.

Eaton, J., and H.S. Rosen, 1980, Taxation, human capital, and uncertainty, American Economic Review, 70, 705-715.

Heckman, J.J., 1976, A life-cycle model of earnings, learning, and consumption, Journal of Political Economy, 84, S9-S44.

Heckman, J.J., A. Layne-Farrar, and P. Todd, 1996, Human capital pricing 
equations with an application to estimating the effect of schooling quality on earnings, Review of Economics and Statistics, 78, 562-610.

Jacobs, B., 2005, Optimal income taxation with endogenous human capital, Journal of Public Economic Theory, 7, 295-315.

Jacobs, B., 2007, Optimal redistributive tax and education policies in general equilibrium, CESIFO WP 2162.

Jacobs, B., and A.L. Bovenberg, 2008, Optimal taxation of human capital and the earnings function, CESIFO WP. 2250.

Jacobs, B., D. Schindler, and H. Yang, H., 2009, Optimal taxation of risky human capital, CESIFO W.P. NO. 2529.

Krueger, A.B., and M. Lindhal, 2000, Education for growth: Why and for whom?, Journal of Economics Literature, 39, 1111-1136.

Lloyd-Ellis, H., 2000, Public education, occupational choice, and the growth inequality relationship, International Economic Review, 41, 171-202.

Lucas, R.E., 1988, On the mechanics of economic development, Journal of Monetary Economics, 22, 3-42.

Mendolicchio, C., D. Paolini and T. Pietra, 2008, Investments in education in a random matching model, CRENoS WP 2008/09.

OECD, 2008, Education at a Glance 2008. OECD Indicators, OECD, Paris.

Roy, A., 1951, Some thoughts on the distribution of earnings, Oxford Economic Papers, 3, 135-146.

Sahin, A., 2004, The incentive effects of higher education subsidies on student effort, Federal Reserve Bank of New York, Staff Report 192.

Sattinger, M., 1993, Assignment models of the distribution of earnings, Journal of Economic Literature, 31, 831-880.

Sattinger, M., 2003, A search version of the Roy model, mimeo.

Schindler, D., and B. Weigert, 2008, Insuring Educational Risk: Opportunities versus Income, CESifo WP 2348.

Schindler, D., and B. Weigert, 2009. Educational and Wage Risk: Social Insurance vs. Quality of Education, CESifo WP 2513.

$\mathrm{Su}, \mathrm{X} ., 2004$, The allocation of public funds in a hierarchical educational system, Journal of Economic Dynamics and Control, 28, 2485-2510. 